\title{
An Extended Car-Following Model in Connected and Autonomous Vehicle Environment: Perspective from the Cooperation between Drivers
}

\author{
Shenzhen Ding $\mathbb{D}^{1},{ }^{1}$ Xumei Chen $\mathbb{D D}^{1,2}{ }^{1,2}$ exin Fu, ${ }^{1}$ and Fei Peng ${ }^{1}$ \\ ${ }^{1}$ Key Laboratory of Transport Industry of Big Data Application Technologies for Comprehensive Transport, Ministry of Transport, \\ Beijing Jiaotong University, Beijing 100044, China \\ ${ }^{2}$ Xuchang University, Henan Province, Xuchang 461000, China
}

Correspondence should be addressed to Xumei Chen; xmchen@bjtu.edu.cn

Received 22 July 2021; Accepted 17 September 2021; Published 13 October 2021

Academic Editor: Francesco Galante

Copyright $(2021$ Shenzhen Ding et al. This is an open access article distributed under the Creative Commons Attribution License, which permits unrestricted use, distribution, and reproduction in any medium, provided the original work is properly cited.

The development of connected and autonomous vehicle (CAV) technology has received increasing attention in recent years. Although car-following behavior in mixed traffic with CAVs and human-driven vehicles (HDVs) is a core component of microscopic traffic simulation, intelligent traffic systems, etc., the current study of car-following behavior in mixed traffic has some limitations. Furthermore, actual data do not support its applicability to the Chinese traffic environment. To address this gap, this paper designs and organizes a car-following experiment in mixed traffic in Beijing, extracts the trajectory data of CAVs and HDVs based on video recognition, and reconstructs the extracted trajectory data using the Lagrangian theory and Kalman filter theory to ensure the accuracy of the data. Based on this data set, this paper develops an extended car-following model. The model considers the cooperation between drivers by reformulating the prospect theory (PT). The root mean square percentage error (RMSPE) is selected to calibrate and validate the parameters of the proposed model, and the results show that there is significant heterogeneity between CAVs and HDVs in mixed traffic, and the proposed model captures this heterogeneity well. The model presented in this paper provides theoretical support for microscopic traffic simulation in mixed traffic.

\section{Introduction}

Traffic congestion has been a hot issue for decades and will remain so in the future. Generally, traffic congestion causes oscillations and stop-and-go waves, which are a nuisance to drivers and result in more accidents and lower traffic efficiency. Many fascinating traffic flow phenomena, such as hysteresis, traffic breakdown, and capacity drop, result from the complexity and randomness of driving behavior [1]. To better alleviate traffic congestion and control traffic flow, it is necessary to understand the mechanism of these phenomena. However, our understanding of traffic flow is still not comprehensive and is even somewhat ambiguous [2].

In addition, understanding and exploring these human behaviors are just as important in the field of CAVs. At present, despite the enormous investments in CAV technologies that the automotive industry has made to compete in this potential market, it will still require decades for CAVs to be fully deployed [3]. It is a fact that mixed traffic, including CAVs and HDVs, will appear and last for a long time. In the second and third level automatic driving stages, although the driver does not need to perform driving operations, they need to monitor the road environment and the autonomous driving system and respond to takeover requests from the system in time $[4,5]$. How will these two types of vehicles, CAVs and HDVs, operate together in mixed traffic? How can we better understand the driving behavior in mixed traffic and thus improve traffic conditions? A model of the collaborative interaction between two types of vehicles is one of the many essential tools.

As one of the microscopic traffic flow models, the carfollowing model has been developed for many decades 
because of its ability to control the longitudinal part of the traffic flow. During the following process, novice drivers feel very nervous due to a lack of confidence or inaccurate judgment of the relative speed and distance between the cars in front and behind, which causes drivers to have very high stress and thus affects their following behavior. Skilled drivers may feel bored during car-following, but their rich driving experience allows them to drive steadily behind the preceding vehicle [6]. For different types of drivers, driving behaviors will vary during the following process, and the study of these behavioral characteristics is essential for microscopic traffic flow. The first car-following model, the Pipes model, was proposed in 1953 [7]. Since then, numerous car-following models have been proposed, reflecting the evolution of the study of traffic flow from different perspectives. These models mainly include the Gazis-Herman-Rothery model [8], the intelligent driver model [9], the optimal velocity model [10], the Gipps model [11], and the Wiedemann model [12]. These models describe the driving behavior during the car-following from different perspectives, such as stimulus-response, safety-distance, optimal velocity, and psycho-physical. Among them, many methods and conclusions have been proposed to enhance traffic flow stability, improve safety, and alleviate traffic congestion [13]. However, the analysis of vehicle interactions cannot be separated from human factors, which are often neglected in previous studies. The introduction of human factors complicates the interaction between vehicles [14]. An accurate description of the human-like collaborative relationship between cars during the following process is conducive to understanding the operation rules of heterogeneous traffic flow and then to implement traffic management and control measures more effectively.

Recently, emerging automated vehicle (AV) technologies push the study of driver behavior to a new stage, especially car-following behavior. As the precursor of $\mathrm{AV}$ technology, the adaptive cruise control (ACC) has been very common. It has been more than 20 years since the advent of the first vehicle equipped with ACC technology [15]. Moreover, the ACC technology is likely to be the primary component for longitudinal control of higher-level AVs in the future [16]. Therefore, it is critical to understand the changes brought about by vehicles equipped with ACC systems compared with traditional HDVs. Although vehicles equipped with ACC systems have become common, only a few studies have been conducted on the driving behavior of ACC vehicles based on experimental field data. Several studies have been conducted in the literature on the stability of ACC systems [17-19]. Notably, in recent years, pioneering work has been done to understand the behavior of ACC, and more sophisticated control experiments have been conducted. Specifically, Gunter et al. have undertaken a set of car-following experiments to collect empirical data. They calibrated an optimal velocity car-following model in their research and analyzed the string stability for both calibrated models based on the observed data [20]. Moreover, the Joint Research Centre of the European Commission has conducted a set of ACC experiments, and some basic features of ACC have already been revealed [21]. However, ACC systems make car-following decisions that depend only on the preceding vehicle's information, weakening the connectivity of cars equipped with the ACC system. Compared with the ACC system, the cooperative adaptive cruise control (CACC) system makes car-following decisions with more information from either a single vehicle or multiple vehicles using connectivity technologies [22]. The CACC system allows vehicles to follow in a platoon by extra layers of communication and automation [23]. As one of the most popular CAV technology applications, scholars have paid a significant amount of attention to the CACC system [24-27]. However, the research on CACC systems has focused on what benefits CAVs may bring to traffic networks, with little consideration of the impact of CACC systems on vehicles not equipped with the CAV technology (HDVs). So, it is particularly important to comprehensively consider the cooperative relationship between CAVs and HDVs in mixed traffic to conduct interaction analysis and achieve the optimal system.

A common and perhaps the most critical gap identified in the above studies is the insufficient consideration of human factors during the following process. The cooperation between human drivers (or between a human driver and a CAV with a higher autopilot system) significantly affects the car-following operation. In addition, a few studies directly used existing car-following models to simulate the behavior of vehicles in mixed traffic without examining the applicability of the models, either for CAVs or for HDVs. Further, most previous studies have used the vehicle trajectory data from driving simulators and numerical simulations, and they employed trajectory data of HDVs to calibrate the model. Still, these data are not representative of real-world vehicle trajectory data in connected and autonomous vehicle environment. The car-following behavior of both CAVs and HDVs has changed in connected and autonomous vehicle environment. The traditional car-following model is no longer sufficient to describe their driving behavior. Therefore, how can we obtain accurate and credible vehicle trajectory data in connected and autonomous vehicle environment? How can we take human factors into account when modeling car-following behavior in mixed traffic? These questions are still to be explored.

Thus, the paper focuses on modeling car-following behavior in connected and autonomous vehicle environment by incorporating human factors, i.e., cooperation between drivers. The well-known PT describes the cooperation between drivers in this paper. Three variables-speed difference, acceleration, and distance headway-are utilized to model the car-following behavior of vehicles. Also, we designed and conducted a car-following experiment in connected and autonomous vehicle environment to obtain the vehicle trajectory data required for the calibration and validation of the model. In the experiment, three Tesla vehicles, equipped with Level 2 autonomous driving functions, completed the designed driving operations on the test road section. Using the Lagrangian theory and Kalman filter theory, we developed a vehicle trajectory reconstruction method to reconstruct the extracted data from the collected videos. 
The remainder of the paper is structured as follows. The following section (Section 2) is about data collection, which mainly explains the experimental and data reconstruction method. Section 3 presents the analysis of cooperation for car-following behavior in mixed traffic. Section 4 describes the methodologies to model car-following behavior in connected and autonomous vehicle environment based on PT. The calibration and verification of the model are provided in Section 5. The last section concludes the paper.

\section{Data Collection and Processing}

High-precision vehicle trajectory data are necessary to support the research of micro-driving behavior in connected and autonomous vehicle environment. However, the vehicle trajectory data acquired based on in-vehicle devices or videos have non-negligible measurement errors that adversely affect the establishment and calibration of microscopic driving models. Thus, a data acquisition experiment was designed and implemented.

In this paper, the experiment was carried out using three Tesla vehicles. Some well-known experiments on mixed traffic were also done with the help of a few test vehicles. For example, the CACC experiments of PATH Laboratory at the University of California, Berkeley. In the study of modeling cooperative and autonomous adaptive cruise control dynamic responses, their experiments were carried out using four production Infiniti M56s (the vehicle equipped with a commercial ACC system) provided by Nissan [28]. Then, we extracted the collected videos to obtain the original vehicle trajectory data. To obtain richer data, a total of 90 minutes of video data were collected for trajectory extraction (greater than 45 minutes in NGSIM), with a granularity of 0.1 seconds. Finally, a two-step reconstruction method was presented by combining the Lagrange algorithm and Kalman filter theory to achieve interpolation replacement and noise reduction of the abnormal data in the data set.

2.1. Experimental Implementation of the Cooperative Adaptive Cruise Control. The video data used for vehicle trajectory extraction in this study were collected on December 27, 2019. Ninety minutes of video data, divided into six segments, were collected every 15 minutes. The collection site was located at Majiabao West Road, Fengtai District, Beijing, with a total length of about $230 \mathrm{~m}$, and the collection method was $4 \mathrm{~K}$ high-definition video. Figure 1 shows the survey location and camera position.

To obtain actual data for mixed traffic, three Tesla vehicles, which can achieve Level 2 autonomous driving, were driven back and forth on Majiabao West Road in Beijing for 1.5 hours. The videos are recorded with a high-resolution camera located on a high building near Majiabao West Road. The autonomous driving functions, including adaptive cruise control, lane keep assistance, and lane departure warning, are active when the drivers enter the test area. To simulate a connected environment, the drivers of the three Tesla vehicles interact via real-time video communication. For example, when a Tesla driver performs a lane-changing operation, the other two Tesla drivers in adjacent lanes are informed through real-time video communication and then cooperate with the driver to complete the operation. In addition, the three Tesla vehicles share speed information in real time. In this way, the experimental vehicles can simulate a CACC scenario. The vehicles could receive speed advice from other vehicles via real-time video communication and then decide whether to perform coordinated acceleration or deceleration. The three Teslas' roofs were marked in purple, green, and yellow to facilitate identifying the test cars in the video, as shown in Figure 2. During trajectory extraction, we combine two methods to improve the accuracy of secondary tracking of Tesla vehicles. On the one hand, we use support vector machines (SVMs) as a classifier to classify the Tesla vehicles and then detect the Tesla vehicle data. The realization of this process uses color filters to generate image features. On the other hand, convolutional neural networks (CNNs) are used to learn the features for the Tesla vehicles, and the accuracy of the Tesla vehicle tracking is improved by controlling the Euclidean distance between different classes of feature vectors.

2.2. Vehicle Trajectory Reconstruction. Professional collaborators have performed video-based extraction of vehicle trajectory data. Vehicle trajectory information extraction includes vital steps, such as coordinate system establishment and conversion, vehicle detection and positioning, vehicle classification, target vehicle tracking, and trajectory information extraction. The vehicle trajectory data obtained by video extraction include a vehicle identification number, recording time, vehicle type, lane number, horizontal coordinate $X$, vertical coordinate $Y$, speed, and acceleration.

The error of vehicle trajectory data extraction based on video is directly related to the sampling frequency of video frames [29]. This error can be divided into two categories. One is the system error based on different detection methods; the other is the random error, i.e., the noisy data generated during the detection process.

For the speed data calculated by the vehicle longitudinal position information, there are two kinds of abnormal values: negative values and extreme values. In addition, the analysis of vehicle acceleration data finds that there is a specific moment when the vehicle speed data are correct. However, the acceleration data are too large, beyond the limit of the physical properties of the vehicle and the bearing capacity of the human body, so this kind of abnormal value also needs to be corrected. The possible causes of system errors include light, occlusion, and other uncontrollable factors. In the process of vehicle trajectory extraction, the unsuccessful recognition of the vehicle position in the image and the abnormal disturbance of the vehicle detection edge can cause system errors. The random error is mainly generated because the original vehicle trajectory data obtained from video extraction will be perturbed up and down around the vehicle's actual position, resulting in obvious noise sequences in the data. This random error is unavoidable, and it will be transferred to the speed and acceleration data. There are many reasons for random errors, such as the external 


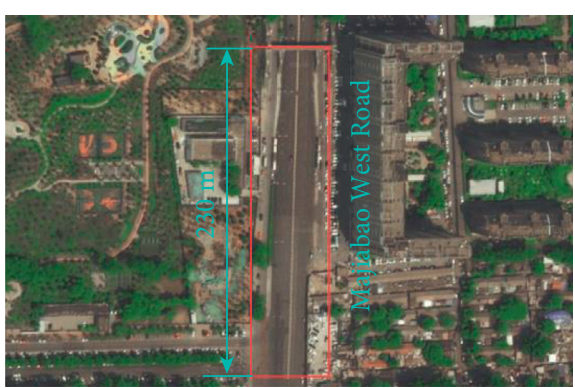

(a)

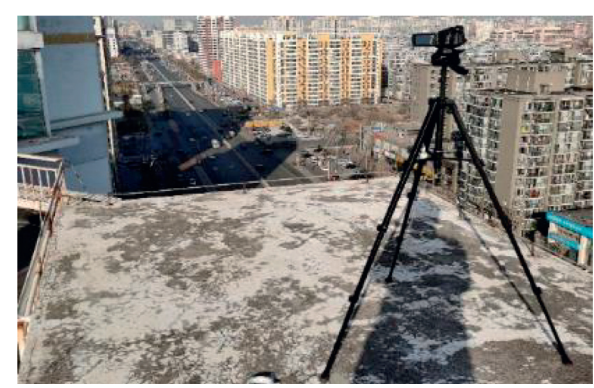

(b)

FIgURE 1: (a) Survey location and (b) camera position.

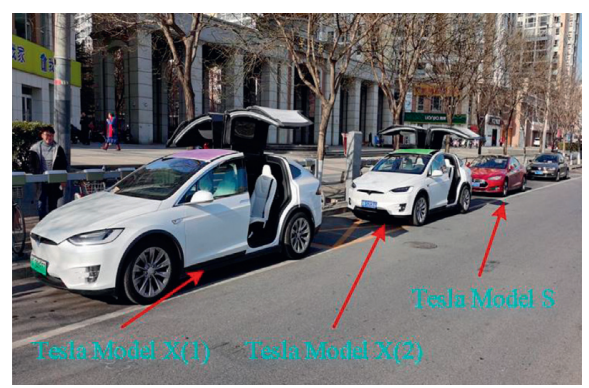

FIgURE 2: Tesla vehicles marked in different colors.

environment, the construction of the instrument itself, and the temporal granularity of the vehicle trajectory extraction. Random errors cannot be eliminated, but they can be filtered by signal filtering techniques, such as Kalman filter techniques [30].

We design a two-step reconstruction method of vehicle trajectory based on the error analysis, as shown in Figure 3. First, according to the physical performance of the vehicle and the human body's tolerance limit, combined with the recorded actual speed data of the experimental vehicles, the speed extremes, negative speed values, and abnormal values of acceleration are identified. The Lagrange interpolation method is used to re-estimate these outliers. Second, due to the random error in video trajectory extraction, the Kalman filter method is used for noise reduction.

Step 1. Vehicle trajectory data correction based on the Lagrangian interpolation method.

Interpolation is the process of solving for the values corresponding to the missing or incorrect discrete points when the series of discrete points and their corresponding values are known [31]. This study uses the Lagrange interpolation method to correct the abnormal values of speed and acceleration. The thresholds of detecting outliers are mainly determined according to the physical performance of the vehicle and the human body's tolerance limit, combined with the actual speed data recorded by the experimental vehicles. In this paper, we adopted detection thresholds of $5 \mathrm{~m} / \mathrm{s}^{2}$ for acceleration and $-8 \mathrm{~m} / \mathrm{s}^{2}$ for deceleration. These thresholds were chosen after large-scale testing [32]. In fact, lower absolute thresholds (close to the physical acceleration of the vehicle) will result in the detection and subsequent removal of a large number of points, and loss of information

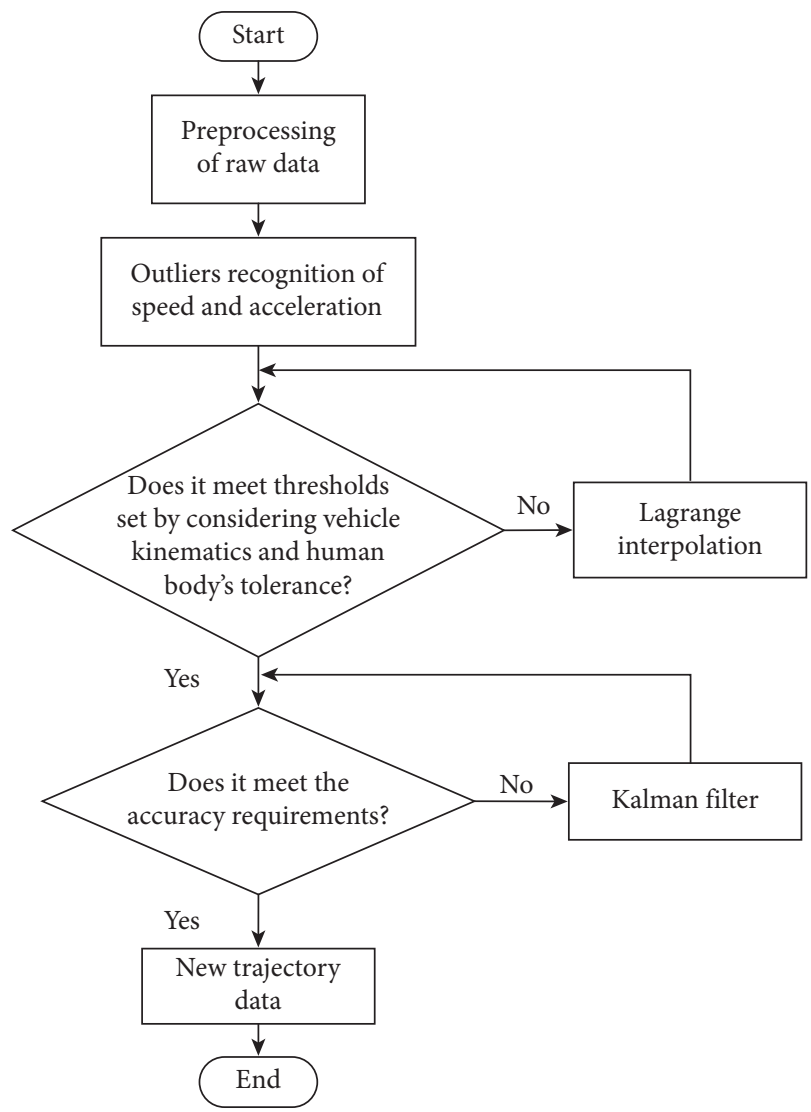

FIGURE 3: Flow chart of vehicle trajectory reconstruction.

about the trajectory trend. Taking the speed correction of the experimental vehicle No. 1 as an example, as shown in Figure 4, by identifying the outliers, the negative speed values, speed extremes, and the vehicle position are effectively corrected.

In this step, the threshold boundary set for identifying abnormal values of speed and acceleration should not be too strict to reduce the impact of the human correction on the original structural characteristics of the vehicle trajectory data.

Step 2. Noise reduction based on Kalman filter method.

After Lagrangian interpolation, the Kalman filter method is used to reduce noise for speed and acceleration. 


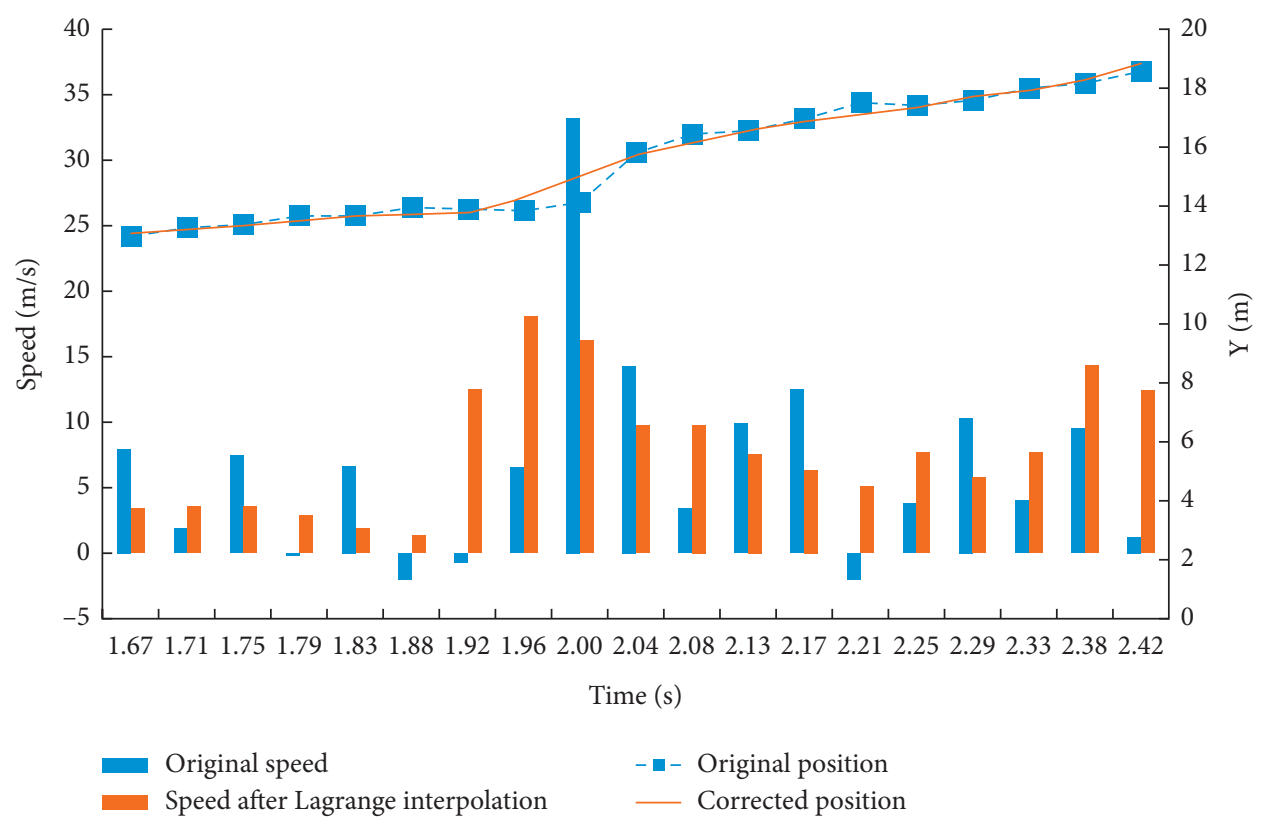

FIgURE 4: Schematic diagram of data correction based on Lagrangian interpolation.

The Kalman filtering is affected by the Kalman gain, which depends on the ratio between the system noise and the observed noise. The two noises are expressed using their respective covariances. The system noise covariance is expressed as $Q$, and the observed noise covariance is expressed as $R$. Since the calculation of the Kalman gain depends on the ratio between $Q$ and $R$, four groups are designed in this study with $R$ as the variable, that is, $Q=0.0001, \quad R=0.001, Q=0.0001, \quad R=0.005, \quad Q=0.0001$, $R=0.01$, and $Q=0.0001, R=0.1$, to compare and analyze the efficiency of the Kalman filtering, as shown in Figure 5.

Figure 5 shows that the filtered curves of different groups display different trends. Group 1 is closest to the original curve, and Group 4 has the smoothest curve after noise reduction. Still, some speed measurements of Group 1 are greater than $70 \mathrm{~km} / \mathrm{h}$ after noise reduction, and the maximum speed value of Group 4 is less than $50 \mathrm{~km} / \mathrm{h}$ after noise reduction, neither of which matches the actual value recorded in the experimental field. The effect of noise reduction for Groups 2 and 3 could not be judged by Figure 5 alone, so this study uses the recorded actual speed data of the experimental vehicles to calculate the percentage error of different speed intervals, as shown in Figure 6. As Figure 6 shows, Group 2 has the best noise reduction effect and the smallest cumulative percentage error, $9.4 \%$. In addition, the percentage error of Groups 4 and 1 after noise reduction is more considerable, presumably due to the under-reduction and overfitting phenomena in the Kalman filter process. So, the ratio between the system noise and the observed noise should fall reasonably within the accuracy requirements and the original data characteristics.

Analysis of the reconstructed trajectory data of Group 2 reveals that the speed values are all within $70 \mathrm{~km} / \mathrm{h}$, which is consistent with the actual value recorded in the experimental field. In addition, the acceleration values of Group 2 also meet the constraints of vehicle dynamics limits and the human body's tolerance, all concentrated between $-3 \mathrm{~m} / \mathrm{s}^{2}$ and $3 \mathrm{~m} / \mathrm{s}^{2}$, as shown in Figure 7 .

\section{Analysis of Cooperation for Car-Following Behavior in Mixed Traffic}

The experiment collects six 15-minute videos. After trajectory extraction and reconstruction, about 821,045 frames of vehicle trajectory data of 9730 vehicles are obtained (1 frame is equal to $0.1 \mathrm{~s}$ ). It is necessary to define the types of car-following behavior to model the car-following behavior of vehicles in mixed traffic. The distributions, such as the speed, acceleration, and distance headway, are analyzed and then used to understand the cooperation between drivers during the following process.

3.1. Different Types of Car-Following Behavior in Mixed Traffic. There are two vehicle types in mixed traffic, CAV and HDV, so a total of four car-following types are derived, as shown in Figure 8. Since CAVs are equipped with automatic driving and communication functions, they can obtain information about the speed and distance of the vehicle in front of them. However, HDVs do not have these functions, so we speculate that their car-following behavior differs from each other.

Figure 8 shows that there are four car-following types in mixed traffic, namely CAVs followed by CAVs, CAVs followed by HDVs, HDVs followed by CAVs, and HDVs followed by HDVs. Twenty pairs of car-following groups ( 5 pairs for each car-following type) are selected from the vehicle trajectory set to study the distribution of the vehicle's speed, acceleration, and distance headway during the following process. Figure 9 shows the vehicle's speed 


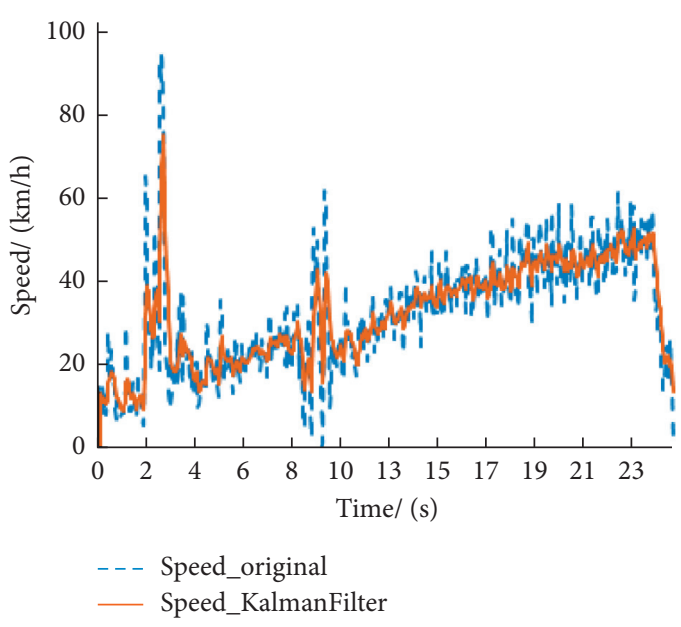

(a)

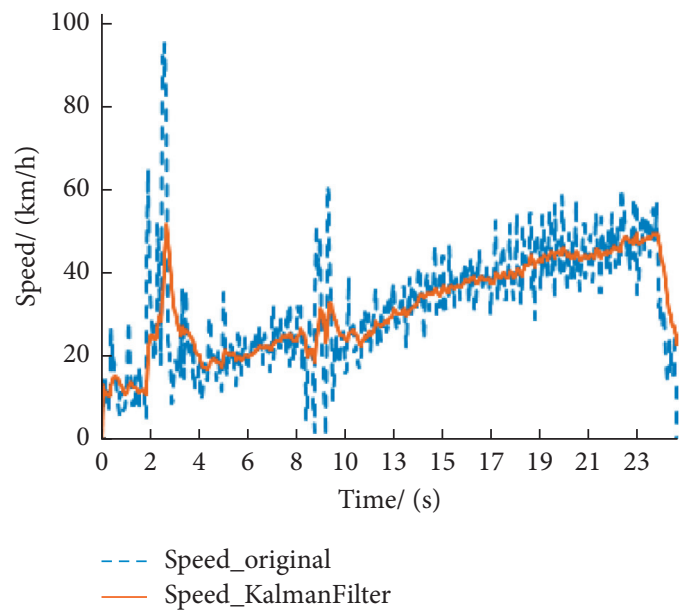

(c)

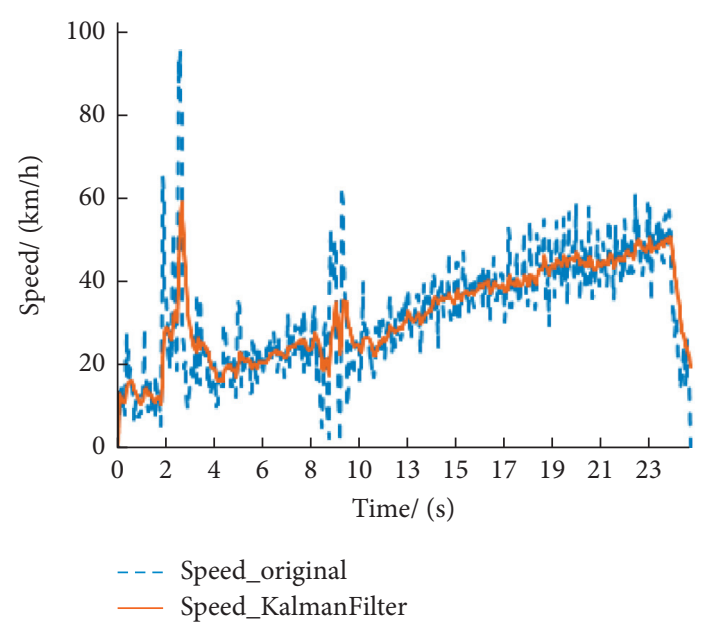

(b)

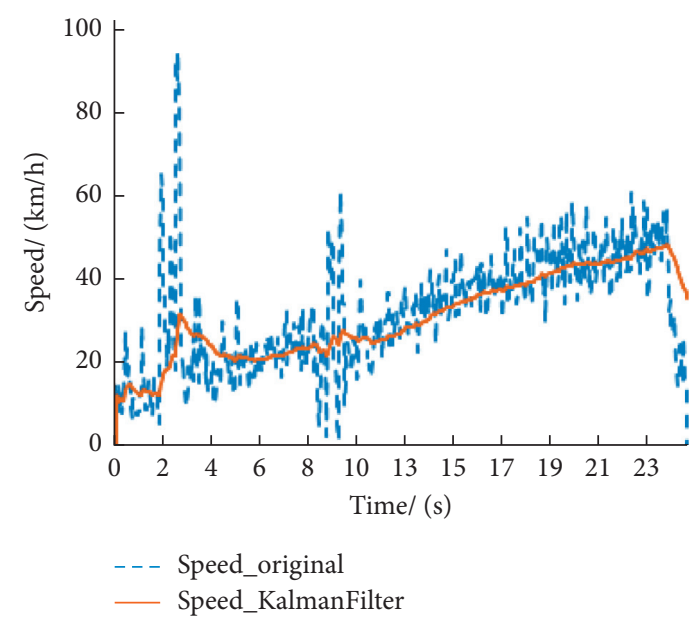

(d)

Figure 5: Comparison of different Kalman filter parameter settings. (a) Group 1; (b) Group 2; (c) Group 3; and (d) Group 4.

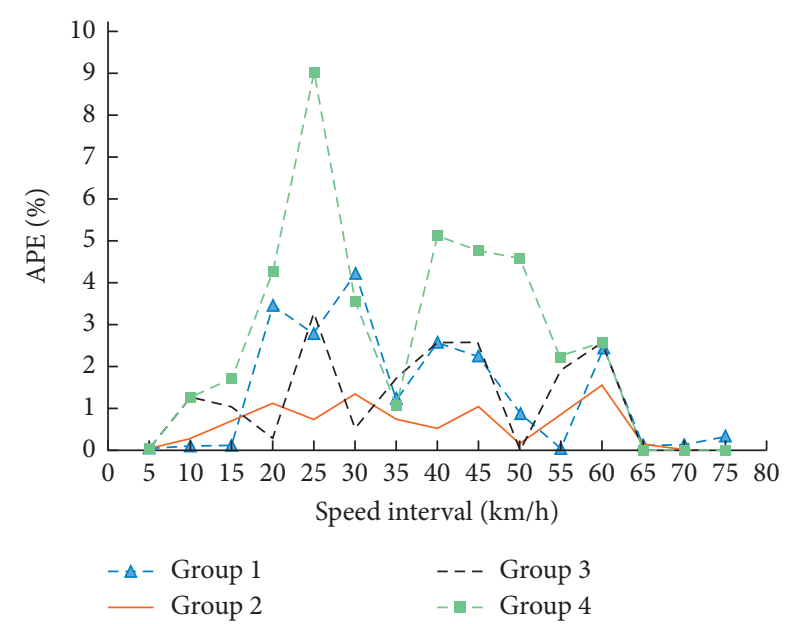

FIgURE 6: Absolute percentage error (APE) for different speed intervals.

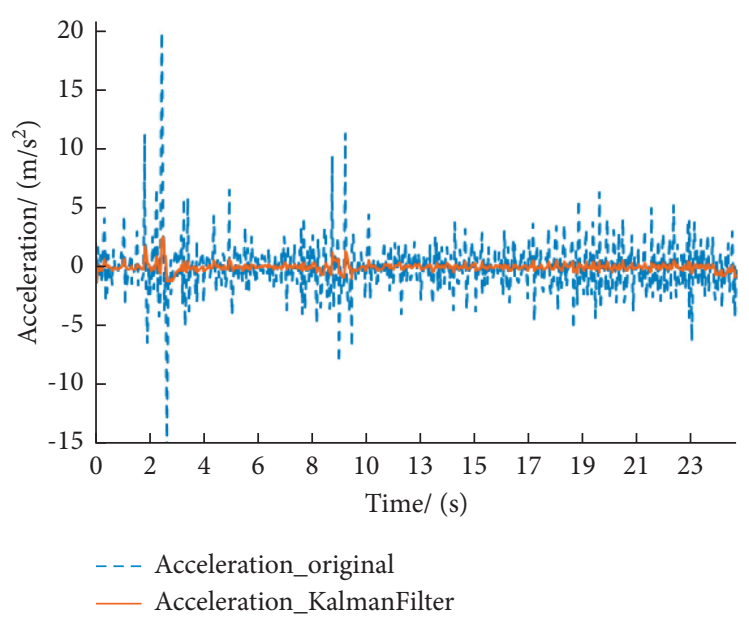

FIgURE 7: Comparison of acceleration before and after Kalman filter for Group 2. 


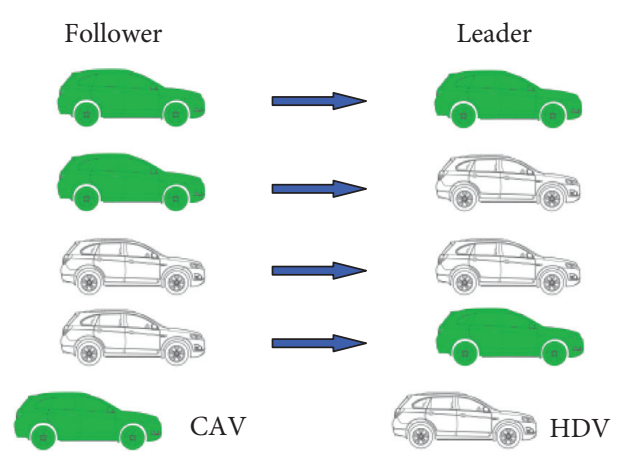

Figure 8: Four car-following types in mixed traffic.

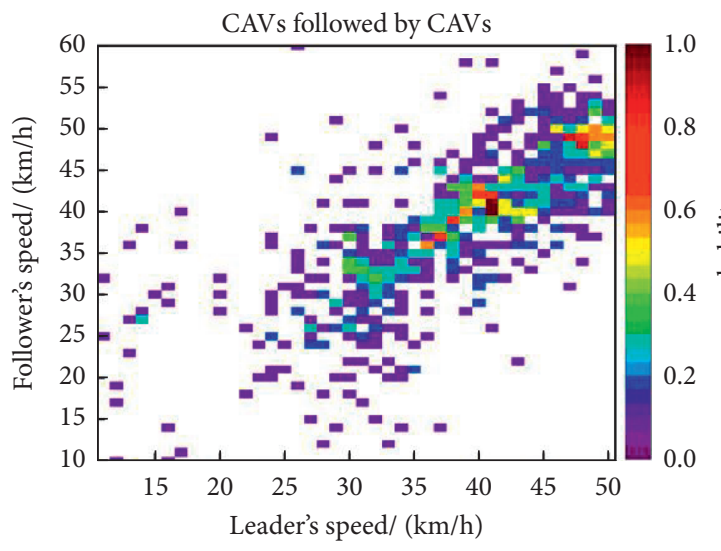

(a)

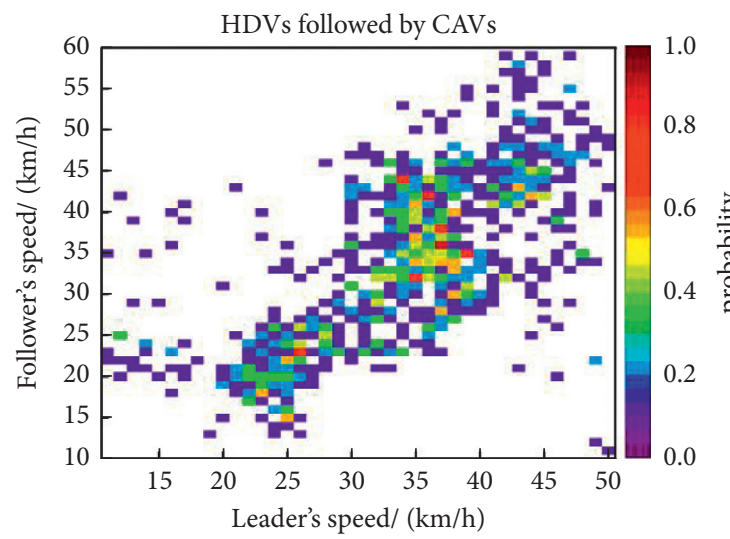

(c)

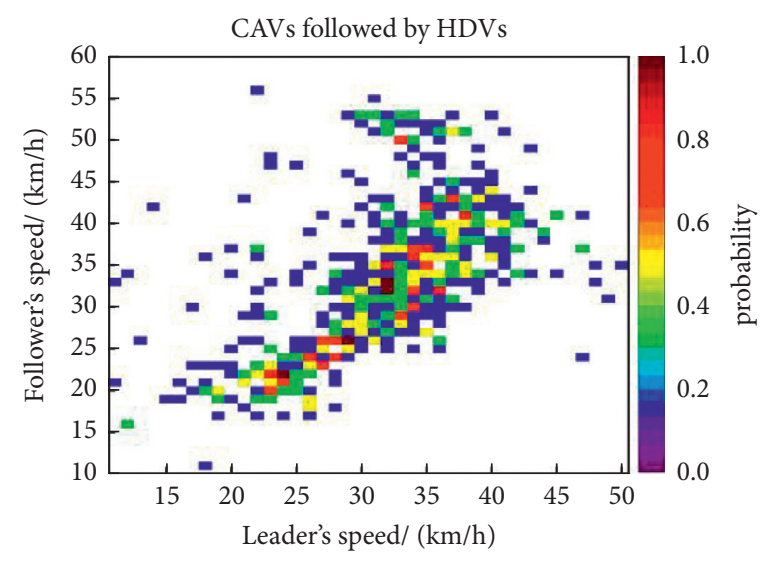

(b)

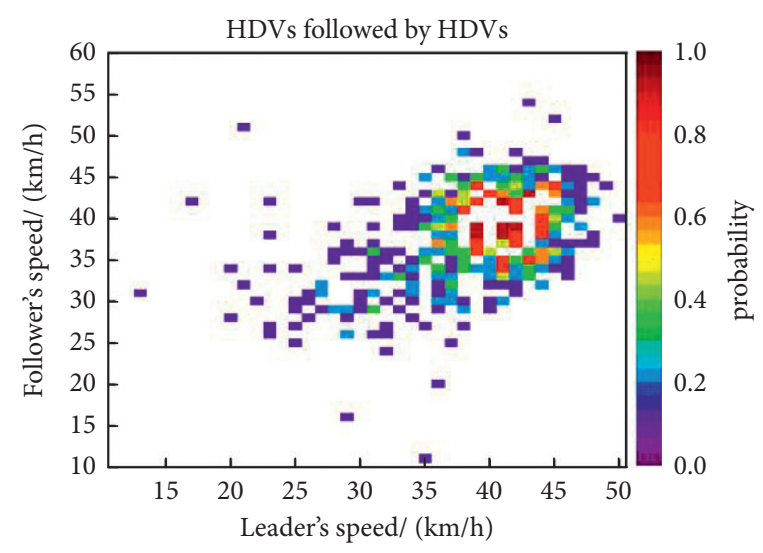

(d)

Figure 9: Vehicle's speed distributions of four car-following types. (a) Type 1; (b) Type 2; (c) Type 3; and (d) Type 4.

distributions of the leader and follower for the different carfollowing types.

As shown in Figure 9, for the first of the four types of carfollowing, when CAVs follow CAVs, the vehicle speed is distributed in the high-speed region. The possible reason is that both vehicles use CAV technologies, and thus the willingness to cooperate between drivers is stronger. The speed distributions of the other three types are similar. There is no communication between the front and rear vehicles for these three car-following types, which affects the car-following behavior of the driver. The distribution relationship between the speed difference and the follower's acceleration for different car-following types appears in Figure 10.

Although the speed distributions are concentrated in the high-speed region when the vehicles in the car-following pairs are both CAVs, the distributions of the speed difference and the follower's acceleration are more dispersed, as shown in Figure 10. Figure 10 also shows that the distributions are more concentrated for the car-following pairs of Types 2 and 4 , which indicates that when the follower is a CAV, the vehicle is more sensitive to the speed change of the front vehicle. Therefore, the acceleration change of the follower is 


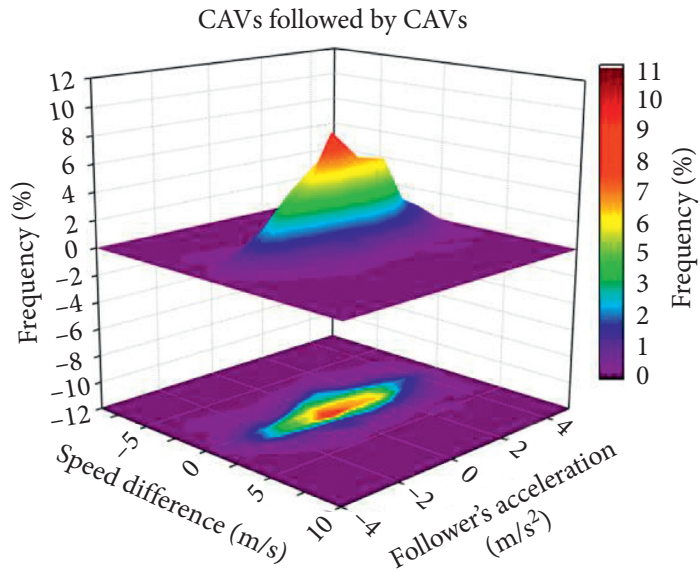

(a)

HDVs followed by CAVs

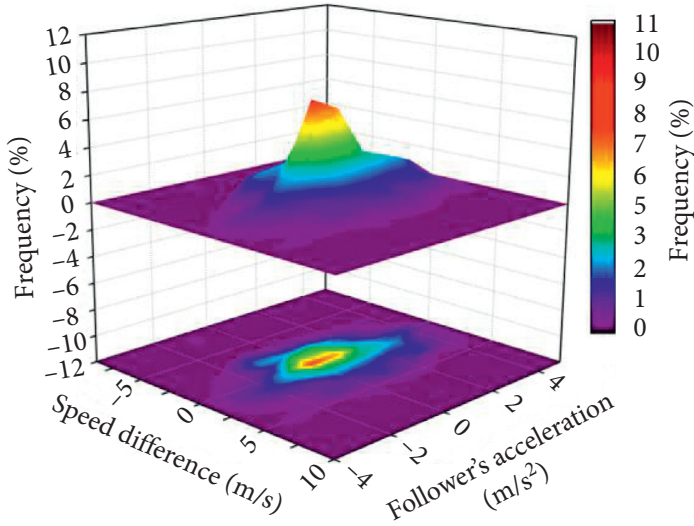

(c)

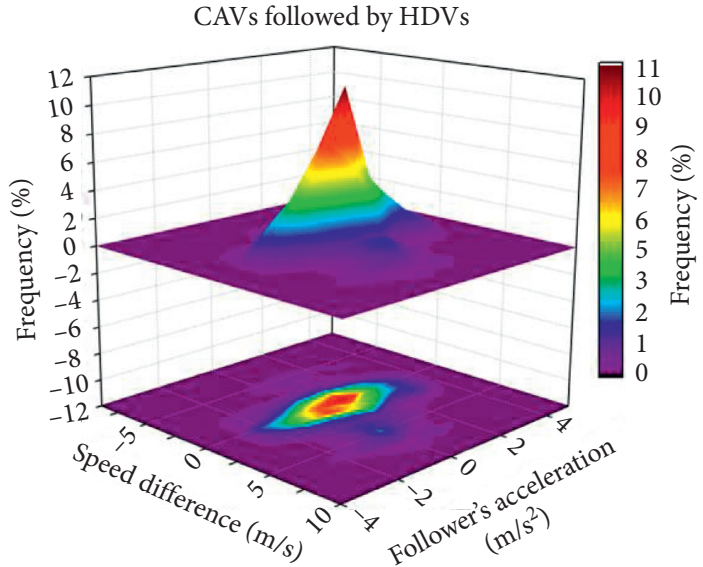

(b)

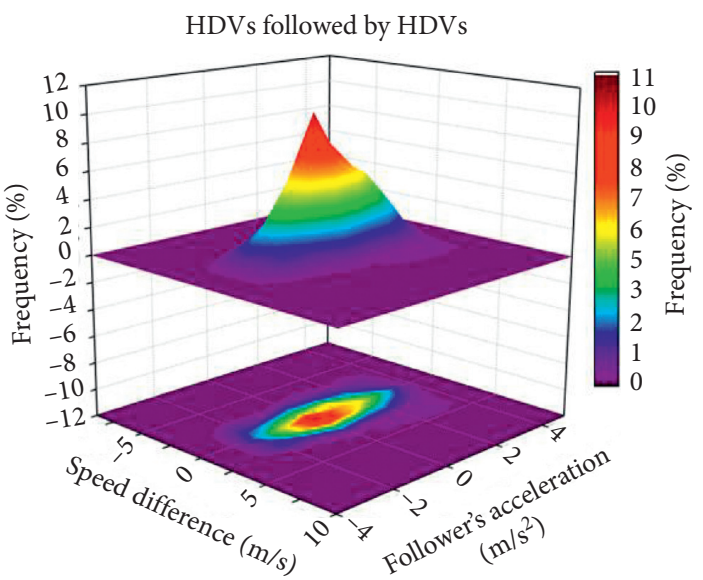

(d)

FIgURE 10: The distribution relationship between the speed difference and the follower's acceleration for different car-following types. (a) Type 1; (b) Type 2; (c) Type 3; and (d) Type 4.

also more sensitive. The situation reverses when the follower is an HDV.

We further analyzed the distance headway distribution when the follower is a CAV or an HDV, respectively, as shown in Figure 11. From Figure 11(a), we can see that, in general, the distance headway distribution is more discrete when the follower is a CAV, but the median value is smaller than that when the follower is an HDV. Further analysis of the distance headway distributions for the four car-following types shows that the second car-following type causes this phenomenon. When both vehicles in the car-following pair are the CAV, the distance headway distribution is more concentrated, and the median value is smaller, which can be seen in Figure 11(b).

3.2. Analysis of Cooperation during the Following Process. In this part, taking the car-following pairs in the data set as an example, the cooperation between vehicles is explained from the variation of speed, headway, and the follower's acceleration. Figure 12 shows the speed variation of the vehicles, where Figures 12(a) and 12(b) shows the case that the follower is a CAV and an HDV, respectively.

Figure 12 shows that the speed variation of the leader and follower displays the same trend during the following process, regardless of whether the follower is a CAV or an HDV, which indicates that the speed cooperation between vehicles does exist during the following process. However, a comparison of Figures 12(a) and 12(b) reveals that the speed change of the follower is smoother when the vehicle is an HDV. Conversely, when the follower is a CAV, the speed variation of the vehicle fluctuates more. To further verify the cooperative relationship between vehicles, Figure 13 compares the variation trends of acceleration and distance headway when a CAV and an HDV are the followers.

Analysis of the variation of the follower's acceleration with the distance headway shows that there is also a cooperative relationship between vehicles during the following process. This relationship is more noticeable when the follower is a CAV because the follower's acceleration is more sensitive to the change of distance headway, as shown in Figure 13(a). 


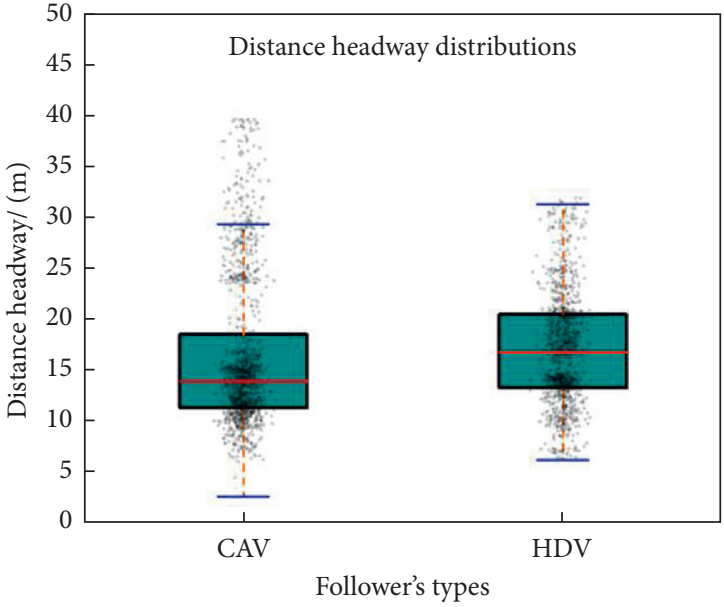

(a)

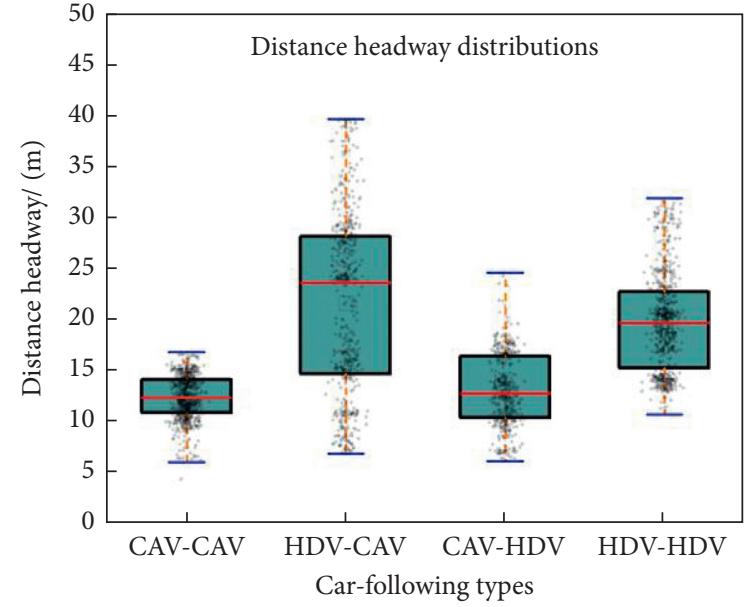

(b)

FIgURE 11: The distance headway distributions. (a) The distributions for different follower's types. (b) The distributions for different carfollowing types.

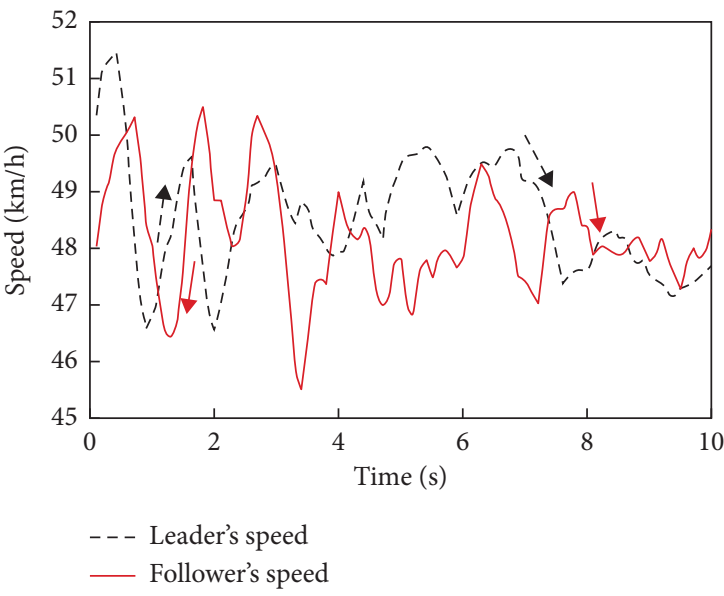

(a)

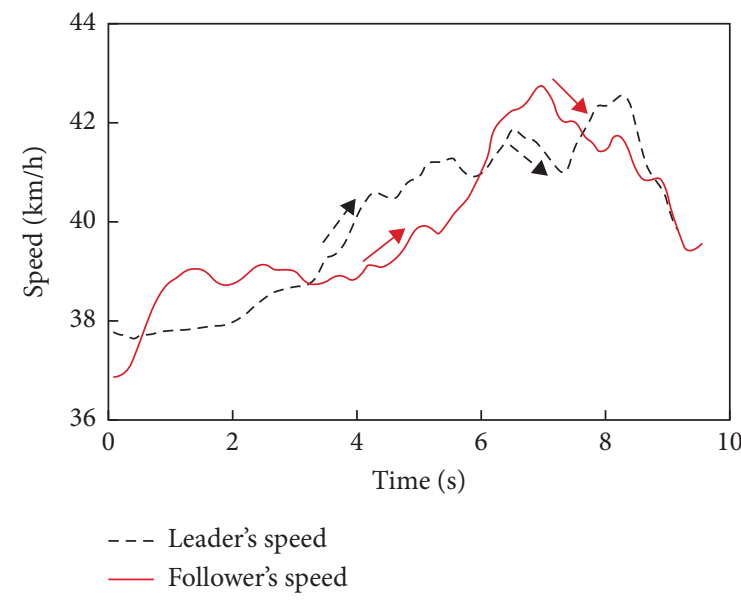

(b)

FIGURE 12: Speed variation during the following process. (a) Speed variation of CAV follower. (b) Speed variation of HDV follower.

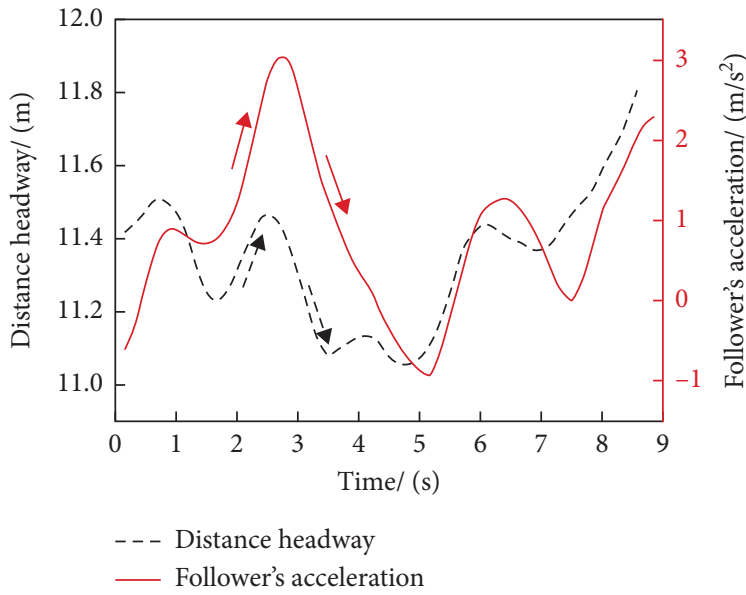

(a)

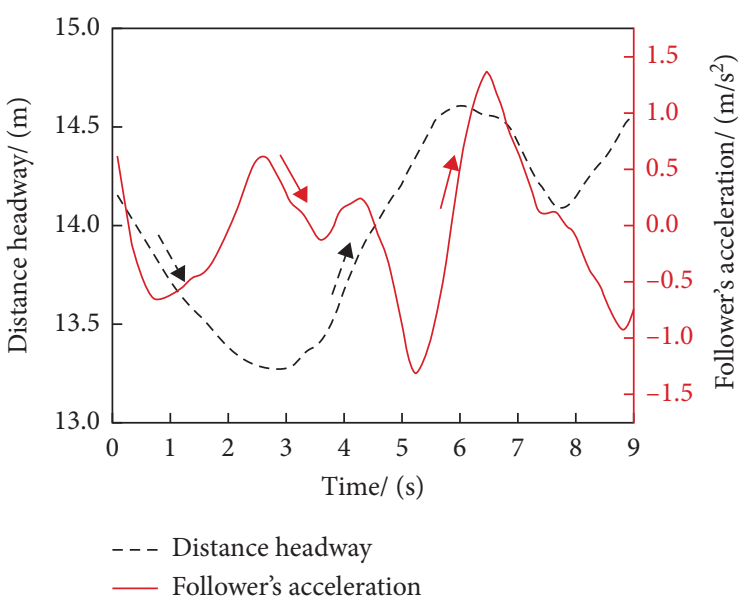

(b)

FIGURE 13: Variation of acceleration and distance headway during the following process. (a) CAV follower. (b) HDV follower. 


\section{Modeling Car-Following Behavior Based on Prospect Theory}

In this section, the cooperation between drivers is measured based on the driver's response to the motion state of the front vehicle, and the cooperation is divided into two levels. A driver's cooperation decision for the state change of the front vehicle (e.g., "leader slowing down") is a classic case of a decision under risk. Decision-making under risk is often modeled by prospect theory (PT) or expected utility theory (EUT). In this paper, PT is used to model the cooperation between drivers because it can describe rational and irrational driving behaviors realistically and consistently, while EUT is best suited for modeling rational decision-makers [14].

4.1. Prospect Theory. Prospect theory, proposed by Daniel Kahneman and Amos Tversky [33], applies psychological research to economics and has made outstanding contributions to the study of human judgment and decisionmaking under uncertainty. In PT, the decision-maker relates the perceived utility of each available choice and selects the most significant perceived utility. In transportation engineering, PT is mainly used to model route choice behavior and is rarely used to model car-following behavior, except for the work by Anshuman et al. [14]. This study uses PT to model driver's compliance in a connected environment. To our knowledge, PT has not been applied to model the cooperation behavior between drivers in a connected and autonomous driving environment.

The options given to the decision-maker are called prospects in $\mathrm{PT}$. The prospects are first formulated to simulate the decision-maker's choices. Then, the utility value of each prospect is calculated. The prospect with the highest utility describes the decision-maker's final choice. A simple prospect includes two outcomes: Gain or Loss expressed by $x$ and Neutral expressed by 0 . Gain means that there are some positive or beneficial supplements to the status of the decision-maker. Loss means that there are some unfavorable supplements to the current status of the decision-maker, and Neutral means that the status of the decision-maker has not changed. The utility related to a simple prospect represented by $U(x, p)$ is the product of the value related to $x$ and $p$ :

$$
U(x, p)=v(x) \cdot w(p) .
$$

The value function $v(x)$ and weight function $w(p)$ can be expressed by equations (2) and (3) [14]:

$$
\begin{aligned}
& v(x)= \begin{cases}x^{\alpha}, & x>0, \\
-\lambda(-x)^{\beta}, & x \leq 0,\end{cases} \\
& w(p)=\frac{p^{\gamma}}{\left(p^{\gamma}+(1-p)^{\gamma}\right)^{1 / \gamma}},
\end{aligned}
$$

where the functions $v(x)$ and $w(p)$ are the values and weights related to the outcome $x$ and probability $p$. The parameters $\alpha, \lambda$, and $\gamma$ control the shape of the PT curve. When the value of $\alpha$ is less than 1 , the value function $v(x)$ will have a concave shape in the gain part and a convex shape in the loss part. When $\lambda>1$, the loss part of the curve is steeper than the gain part, implying that decision-makers are more sensitive to losses. The value of $\gamma$ determines how decision-makers perceive the probability. More specially, when $\gamma$ is equal to 1 , the weight function $w(p)$ becomes linear.

As mentioned by Anshuman et al. in their study, PT has four basic properties, including diminishing sensitivity, reference dependence, loss aversion, and probability weighting. The first three properties are related to the value function, and the last one is related to the weight function. Usually, the sensitivity of decision-makers to gains and losses decreases as the value increases, especially for some large values where the sensitivity value is low. Reference dependence refers to the fact that most people's judgments of gains and losses are often determined by a reference point rather than an absolute value. For example, in a choice between "someone else earns $\$ 50,000$ a year and you earn $\$ 60,000$ a year" and "someone else earns $\$ 80,000$ a year and you earn $\$ 70,000$ a year," most people will choose the former. Loss aversion shows that decision-makers are more sensitive to loss, which is characterized by making the loss part of the value function steeper than the gain part. For example, the pleasure associated with gaining $\$ 100$ cannot offset the pain associated with losing $\$ 100$. Finally, probability weighting shows the characteristics of decisionmakers' perceptions in response to probabilities and is quantified by equation (3). For example, in the face of small probability events, human beings have an ambivalent attitude toward risk. One can be a risk-lover or a risk-averse person, and events with low probability tend to be given high weight by decision-makers.

4.2. Cooperation Modeling Using Prospect Theory. We assumed that all changes in driving behavior due to CAVs technologies are attributable to the cooperative urgency of the driver's current state. Zero cooperation does not lead to a change in the following behavior. In addition, the driver's choice of the level of cooperation is usually dependent on the distance headway when the information is obtained from the vehicle in front. The distance headway reflects the driver's intuitive experience better than the time headway. As the distance headway decreases, the more urgent and easier it is for the driver to cooperate with the vehicle in front, and vice versa. Thus, the cooperation willingness of a driver in response to the change of leader's moving state can be categorized as two levels: low cooperation level and high cooperation level.

The two cooperation levels can be regarded as two prospects, and we use PT to simulate the prospects chosen by the driver. Both prospects are currently treated as simple prospects. In the car-following process, any level of cooperation in response to perceptually obtained information about changes in the driving state of the front vehicle indicates a gain for the driver. In contrast, a complete lack of cooperation suggests a loss. Therefore, we can consider that both prospects consist of gains. Based on the utility formula 
of simple prospect, as shown in equation (1), we define the cooperation level utility as the product of urgency and weight, as shown in equation (4). Urgency indicates the willingness of drivers to cooperate when the state of the front vehicle changes, while weight indicates how drivers perceive different levels of cooperation. The urgency value function is used to calculate the urgency value, and the weighting function is used to calculate the weight.

$$
\text { Cooperation utility }=\text { urgency } \times \text { weight. }
$$

Since the cooperation utility is proportional to the urgency, the greater the urgency, the higher the driver's willingness to cooperate. We assume that the urgency and the observed distance headway have the same inverse relationship as the cooperation. When the driver perceives a change in the motion state of the front vehicle, the smaller the observed distance headway at that time, the greater the driver's willingness to operate collaboratively, and vice versa. In addition, the logistic function is selected for modeling. Considering the nature of PT's urgency function, the urgency value function is expressed by

$$
V\left(d h_{\mathrm{obs}}\right)=\frac{1}{1+e^{\lambda\left(\alpha \cdot d h_{\mathrm{obs}}-1\right)}},
$$

where $d h_{\mathrm{obs}}$ is the observed distance headway, and $\alpha$ and $\lambda$ are the parameters to be calibrated.

The inversely proportional relationship between the urgency value and the observed distance headway is shown in Figure 14. For small and large distance headways, the sensitivity of urgency values decreases (flatter at both ends of the curve), which is consistent with the diminishing sensitivity of PT. In addition, the urgency value function can be used to estimate the distance headway when the urgency value is close to zero and one. When the urgency value is one, it is very urgent, and when the urgency value is zero, it is not necessary to cooperate with the front vehicle. The estimated minimum and maximum distance headway will be used to calculate the weight function.

We assume that each driver has a distance headway range corresponding to each cooperation level. The value of the weight at a particular cooperation level is determined by the probability that the observed distance headway falls within that level distance headway range. Therefore, the weight is higher for this distance headway at high cooperation levels and lower at low cooperation levels. The weighting functions for low and high levels of driver cooperation are also formulated in this way. In addition, the functions reflect the PT properties of probability weighting, i.e., higher weighting for small probability events and lower weighting for significant probability events. Referring to Anshuman et al.'s study [14], the weighting functions are shown as follows.

The low cooperation weighting function is given by equations (6) and (7):

$$
\begin{aligned}
W_{L C}\left(P_{L C}\right) & =\frac{P_{L C}^{\gamma}}{\left(P_{L C}^{\gamma}+\left(1-P_{L C}\right)^{\gamma}\right)^{1-\gamma}}, \\
P_{L C} & =\min \left(\frac{d h_{\mathrm{obs}}}{d h_{\max }}, 1\right) .
\end{aligned}
$$

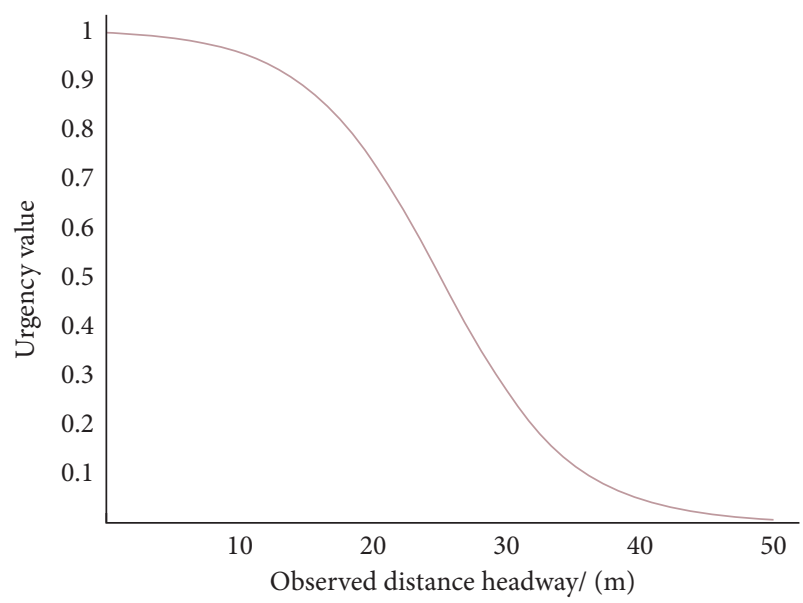

FIgURE 14: Schematic diagram of the urgency value function.

The high cooperation weighting function is given by equations (8) and (9):

$$
\begin{aligned}
W_{H C}\left(P_{H C}\right) & =\frac{P_{H C}^{\gamma}}{\left(P_{H C}^{\gamma}+\left(1-P_{H C}\right)^{\gamma}\right)^{1-\gamma}}, \\
P_{H C} & =\min \left(\frac{d h_{\min }}{d h_{\mathrm{obs}}}, 1\right),
\end{aligned}
$$

where $W_{L C}\left(P_{L C}\right)$ and $W_{H C}\left(P_{H C}\right)$ are the low cooperation weighting function and high cooperation weighting function, respectively. $P_{L C}$ and $P_{H C}$ are the probabilities that an observed distance headway falls in a driver's low and high cooperation ranges. $d h_{\max }$ and $d h_{\min }$ are the distance headways when the values of $V\left(d h_{\mathrm{obs}}\right)$ are close to zero and one, respectively. $\gamma$ is the shape parameter.

Thus, the cooperation utility functions for low and high cooperation levels can be defined by equations (10) and (11), and the final cooperation utility value $U$ is the larger of the low and high utility values, as shown in equation (12):

$$
\begin{aligned}
U_{L C}\left(d b_{\mathrm{obs}}, P_{L C}\right) & =V\left(d b_{\mathrm{obs}}\right) \cdot W_{L C}\left(P_{L C}\right), \\
U_{H C}\left(d b_{\mathrm{obs}}, P_{H C}\right) & =V\left(d b_{\mathrm{obs}}\right) \cdot W_{H C}\left(P_{H C}\right), \\
U & =\max \left(U_{L C}, U_{H C}\right) .
\end{aligned}
$$

4.3. Modeling Car-Following Behavior Considering Cooperation between Drivers. A car-following model is a mathematical description of the influence of the driving environment on driving behavior. The classical car-following model originates from driving dynamics and uses vehicle acceleration to reflect the car-following decision. Based on the classic car-following model of PATH Laboratory, this section models car-following behavior in mixed traffic with HDVs and CAVs from the perspective of cooperation between drivers.

Scholars from the PATH Laboratory at the University of California, Berkeley, have conducted a long-term study on the ACC/CACC car-following model [34]. In the proposed 
car-following model, the acceleration of the following vehicle depends on three components: the acceleration of the front vehicle, the speed difference between the front and rear vehicles, and the error term between the actual distance headway and the desired distance headway. The structure of the car-following model by PATH Laboratory can be expressed as follows:

$$
\begin{aligned}
a_{i} & =k_{0} a_{i-1}+k_{1} e+k_{2}\left(v_{i-1}-v_{i}\right), \\
e & =x_{i-1}-x_{i}-T v_{i},
\end{aligned}
$$

where $a_{i}$ and $a_{i-1}$ represent the acceleration of the rear and front vehicles. $x_{i}$ and $x_{i-1}$ represent the position of the rear and front vehicles. $v_{i}$ and $v_{i-1}$ represent the speed of the rear and front vehicles, respectively. Moreover, $e$ is the difference between the actual distance headway and the desired distance headway. $T$ is the desired time headway. $k_{0}, k_{1}$, and $k_{2}$ are the weights to be calibrated.

Based on the cooperation analysis for car-following behavior in mixed traffic, the car-following behavior of CAVs and HDV s are different. First, CAVs and HDVs have different acceleration, speed, and distance headway distributions. The distributions of acceleration and distance headway for CAVs fluctuate more, and the speed distribution of CAVs also concentrates in the high-speed region. In addition, the cooperative phenomenon between vehicles is more evident when the follower is a CAV than an HDV. Still, the speed and acceleration are more turbulent when the follower is a CAV, probably, because CAVs are more responsive to the front vehicle's state changes than HDVs. This different cooperative nature exhibited by different vehicle types can be characterized by PT, see Section 4.2. In this paper, the extended car-following model in mixed traffic is developed considering the acceleration of the front vehicle, the speed difference between the front and rear vehicles, and the distance headway between the front and rear vehicles. Also, the cooperation willingness of different vehicle types in response to the state changes of the leader is described using PT. Equations (15)-(17) present the mathematical formulations of the proposed model:

$$
\begin{aligned}
a_{n}(t)= & \delta \cdot a_{n-1}(t)+\eta \cdot\left[S_{n}(t)-S_{n}^{*}(t)\right] \\
& +\theta \cdot\left[V_{n-1}(t)-V_{n}(t)\right], \\
S_{n}(t)= & Y_{n-1}(t)-Y_{n}(t), \\
S_{n}^{*}(t)= & S_{0}+\left[1+U\left(d h_{\mathrm{obs}}\right)\right] \cdot T V_{n}(t)+\frac{V_{n}(t) \cdot \Delta V_{n}(t)}{2 \sqrt{a b}},
\end{aligned}
$$

where $a_{n}(t)$ and $a_{n-1}(t)$ are the accelerations of the $n^{\text {th }}$ and $(n-1)^{\text {th }}$ vehicles at moment $t . S_{n}(t)$ is the distance headway of the $n^{\text {th }}$ vehicle at moment $t . S_{n}^{*}(t)$ is the desired distance headway of the $n^{\text {th }}$ vehicle at moment $t . V_{n}(t)$ and $V_{n-1}(t)$ are the speeds of the $n^{\text {th }}$ and $(n-1)^{\text {th }}$ vehicles at moment $t$. $Y_{n}(t)$ and $Y_{n-1}(t)$ are the positions of the $n^{\text {th }}$ and $(n-1)^{\text {th }}$ vehicles at moment $t$. Moreover, $S_{0}, T, a, b, V_{n}(t)$, and
$U\left(d h_{\text {obs }}\right)$ are the standstill distance, desired time headway, maximum acceleration, desired deceleration, speed difference between vehicles, and cooperation utility value, respectively. $\delta, \eta$, and $\theta$ are the parameters to be calibrated.

\section{Calibration and Verification Methodology}

In this paper, RMSPE of distance headway, mentioned by many studies $[13,35]$, is used as the objective function for parameter calibration:

$$
\mathrm{RMSPE}=\sqrt{\frac{1}{n} \cdot \frac{\sum_{i=1}^{n}\left(s_{i}^{\mathrm{sim}}-s_{i}^{\mathrm{act}}\right)^{2}}{\sum_{i=1}^{n}\left(s_{i}^{\mathrm{act}}\right)^{2}}}
$$

where RMSPE of distance headway as the measure of model performance denotes the objective function, $n$ is the number of observations, and $s_{i}^{\text {sim }}$ and $s_{i}^{\text {act }}$ are the $i^{\text {th }}$ simulated distance headway and actual distance headway, respectively.

The genetic algorithm (GA) is used to solve the objective function, and each parameter of GA is set as follows: the population size, the maximum number of generations, the number of stall generations, and the function tolerance are set to $100,300,100$, and $1 e-6$. If the change is less than the function tolerance, the algorithm will stop, and the algorithm finds a different solution in each optimization run. The optimization is repeated ten times for each driver to obtain a solution closer to the global optimum, and the set of parameters with the smallest RMSPE is selected. In addition, to improve the computational tractability of the genetic algorithm optimization process, the paper sets the upper and lower bounds for the parameters of the extended car-following model, as shown in Table 1.

In the parameter calibration, the performance of the proposed model calibration method is tested using the so-called "synthetic data" generated from each driver's trajectory data, as suggested by Punzo et al. [36]. For example, the parameters in the extended car-following model are set to $\alpha=0.3, \lambda=5.5$, $\gamma=0.55, \delta=2.5, \eta=2.5, \theta=2.5, S_{0}=0.55, T=2.5, a=2.5$, and $b=2.5$, and synthetic car-following data are generated. Then, a calibration process is implemented to derive the best model parameters based on the set calibration accuracy.

As mentioned earlier, there are four car-following types in mixed traffic. In particular, the car-following behavior changes significantly whether the rear vehicle is a CAV or an HDV. Therefore, the paper performs model calibration and verification for the rear vehicle as a CAV or as an HDV. We select twenty leader-follower pairs from the data set, including ten pairs of CAV followers and ten pairs of HDV followers. Nine out of ten pairs in each group are used for calibration, and the remaining one is used for calibration. In addition, in order to retain generality, twenty groups of initial parameter data are selected for each leader-follower pair.

\section{Results and Discussions}

Table 2 shows the results of the parameter calibration for a leader-follower pair, and it only offers the calibration 
TABLE 1: Parameter bounds in optimization.

\begin{tabular}{lcc}
\hline Parameters & Definition & Bounds \\
\hline$\alpha$ & Parameter of the urgency value function & {$[0.1,0.5]$} \\
$\lambda$ & Parameter of the urgency value function & {$[1,10]$} \\
$\gamma$ & Parameter of the weighting function & {$[0.1,1]$} \\
$\delta$ & Weight of acceleration & {$[0.1,5]$} \\
$\eta$ & Weight of distance headway & {$[0.1,5]$} \\
$\theta$ & Weight of speed difference & {$[0.1,5]$} \\
$S_{0}$ & Standstill distance & {$[0.1,10]$} \\
$T$ & Desired time gap & {$[0.1,5]$} \\
$a$ & Maximum acceleration & {$[0.1,5]$} \\
$b$ & Desired deceleration & {$[0.1,5]$} \\
\hline
\end{tabular}

TABle 2: Parameter calibration results for a leader-follower pair.

\begin{tabular}{lcccccccccc}
\hline$\alpha$ & $\lambda$ & $\gamma$ & $\delta$ & $\eta$ & $\theta$ & $S_{0}$ & $T$ & $a$ & $b$ & RMSPE (\%) \\
\hline 0.33 & 9.18 & 0.84 & 3.83 & 3.80 & 4.57 & 0.70 & 1.38 & 3.96 & 4.90 & 2.17 \\
0.20 & 3.73 & 0.67 & 2.79 & 1.59 & 4.96 & 9.15 & 3.85 & 1.15 & 1.71 \\
0.49 & 2.10 & 0.30 & 2.40 & 2.60 & 3.66 & 6.72 & 1.43 & 4.05 & 4.92 \\
0.36 & 8.01 & 0.36 & 3.34 & 2.28 & 4.98 & 2.16 & 4.18 & 4.90 & 3.04 & 2.28 \\
0.45 & 5.35 & 0.45 & 1.59 & 0.70 & 3.11 & 7.50 & 2.43 & 2.53 & 0.70 & 2.10 \\
$\mathbf{0 . 1 8}$ & $\mathbf{7 . 7 2}$ & $\mathbf{0 . 9 0}$ & $\mathbf{1 . 4 1}$ & $\mathbf{1 . 9 6}$ & $\mathbf{4 . 0 0}$ & $\mathbf{5 . 1 3}$ & $\mathbf{2 . 0 9}$ & $\mathbf{1 . 8 3}$ & $\mathbf{2 . 1 1}$ \\
0.21 & 5.92 & 0.91 & 2.95 & 0.71 & 2.12 & 6.54 & 4.20 & 3.93 & 2.46 & $\mathbf{1 . 9 2}$ \\
0.45 & 1.76 & 0.69 & 2.00 & 2.17 & 4.99 & 0.38 & 0.54 & 3.79 & 0.99 & 2.02 \\
0.48 & 4.03 & 0.36 & 2.13 & 1.40 & 3.66 & 1.86 & 2.05 & 2.83 & 1.74 & 2.44 \\
0.16 & 9.63 & 0.53 & 2.45 & 1.54 & 4.81 & 4.46 & 4.54 & 3.95 & 1.89 & 2.50 \\
\hline
\end{tabular}

Bold values represent the optimal value of each parameter and the minimum RMSPE value.

results corresponding to the minimum error in each set of initial values. The minimum calibration error of $1.92 \%$ is obtained for the 6th set of initial values, which is also the final calibration error for this leader-follower pair. Figure 15 shows the distribution of the parameter calibration errors.

Figure 16 shows the distributions of the calibrated parameter values in the cooperation utility. The figure also shows that, for CAVs, the value of $\gamma$ is larger than that for HDVs. $\gamma$ is the parameter in the weighting function, and the larger value means that the willingness of the follower to cooperate is stronger when the driving state of a leader changes.

The distributions of the calibrated parameter values in the desired distance headway appear in Figure 17. The figure shows that the distributions of the maximum acceleration and desired time gap are significantly different. Compared with HDVs, the maximum acceleration value of CAVs is larger. At the same time, the desired time gap is smaller, which leads to a shorter desired distance headway of the CAV, indicating that the CAV is more willing to maintain a smaller distance headway with the vehicle in front.

Figure 18 presents the distributions of the calibrated parameter values of $\delta, \eta, \theta$, which respectively represent the weight of the speed difference, the distance headway, and the leader's acceleration. The figure reveals that the value of $\eta$ is larger for CAVs compared with HDVs. A larger value of $\eta$ means that the CAV's acceleration is more influenced by the distance headway compared with the HDV. In particular, the difference in parameters is

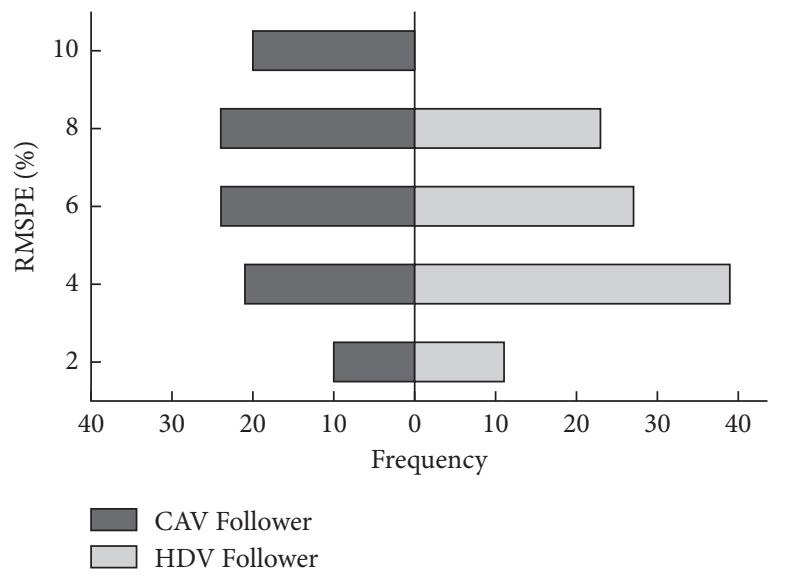

FIgURE 15: Distribution of calibration errors.

attributed to the heterogeneity of CAVs and HDVs. The traffic behavior of CAVs and HDVs in mixed traffic is very different due to the different levels of CAV technology and the involvement of human factors. After determining the optimal values of each parameter, the proposed car-following model is used to calculate the traffic state parameters of the follower. Figure 19 presents the variation of the follower's speed and the variation of the difference between the actual and simulated follower's speed. As shown in Figure 19, the proposed model can well characterize the car-following behavior of the follower, whether it is a CAV or an HDV as a rear vehicle. 

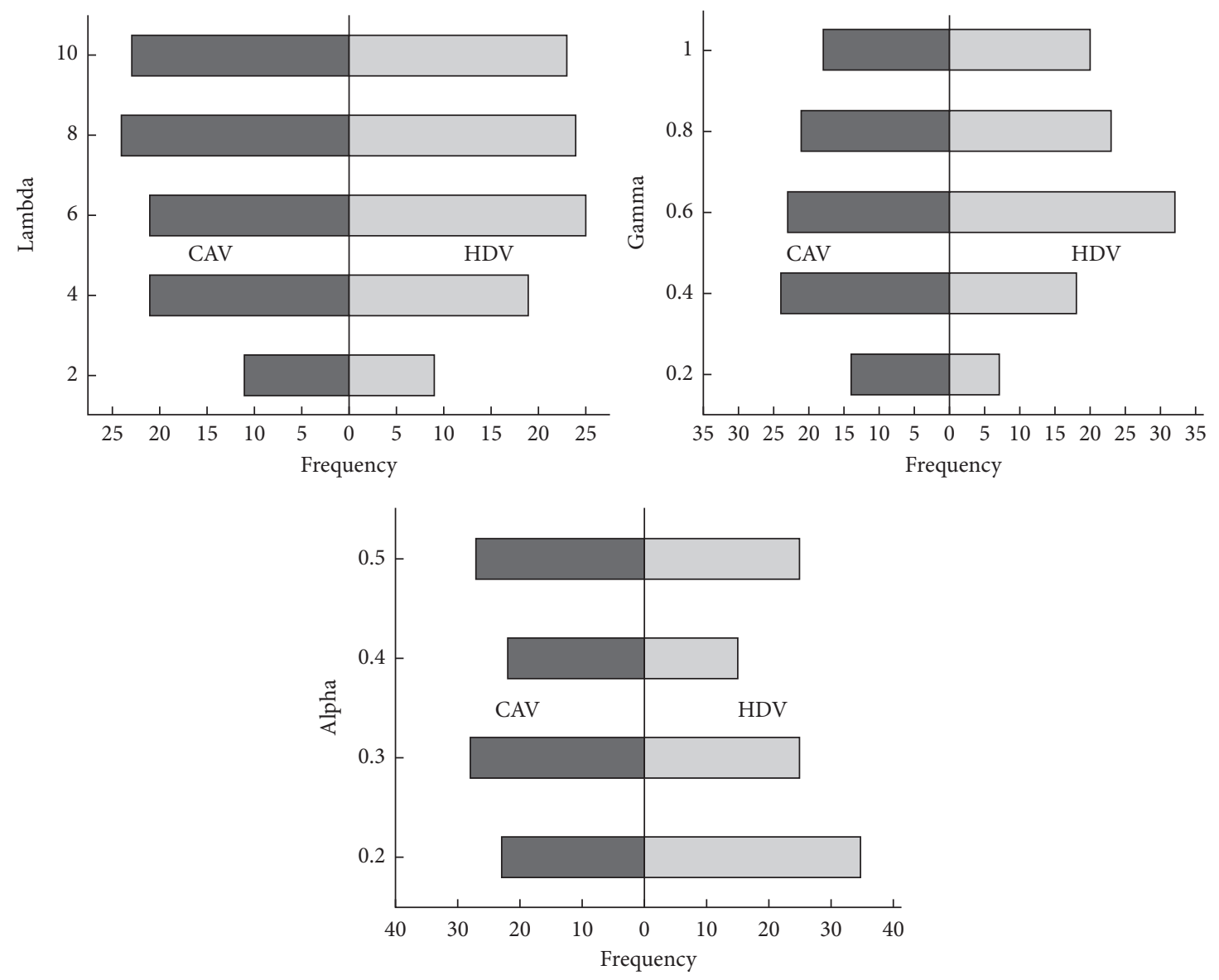

Figure 16: Distributions of the calibrated parameter values in the cooperation utility.
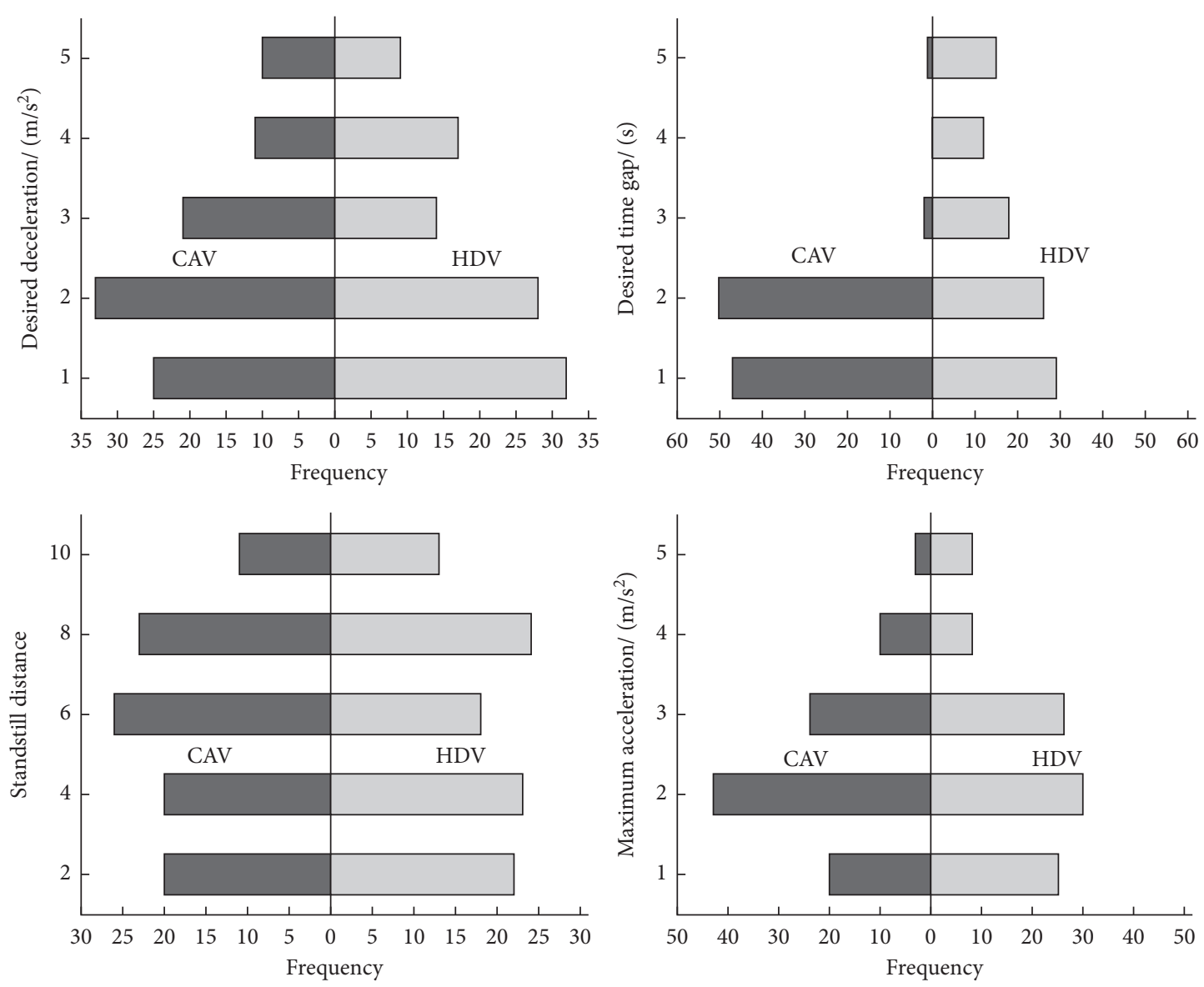

Figure 17: Distributions of the calibrated parameter values in the desired distance headway. 

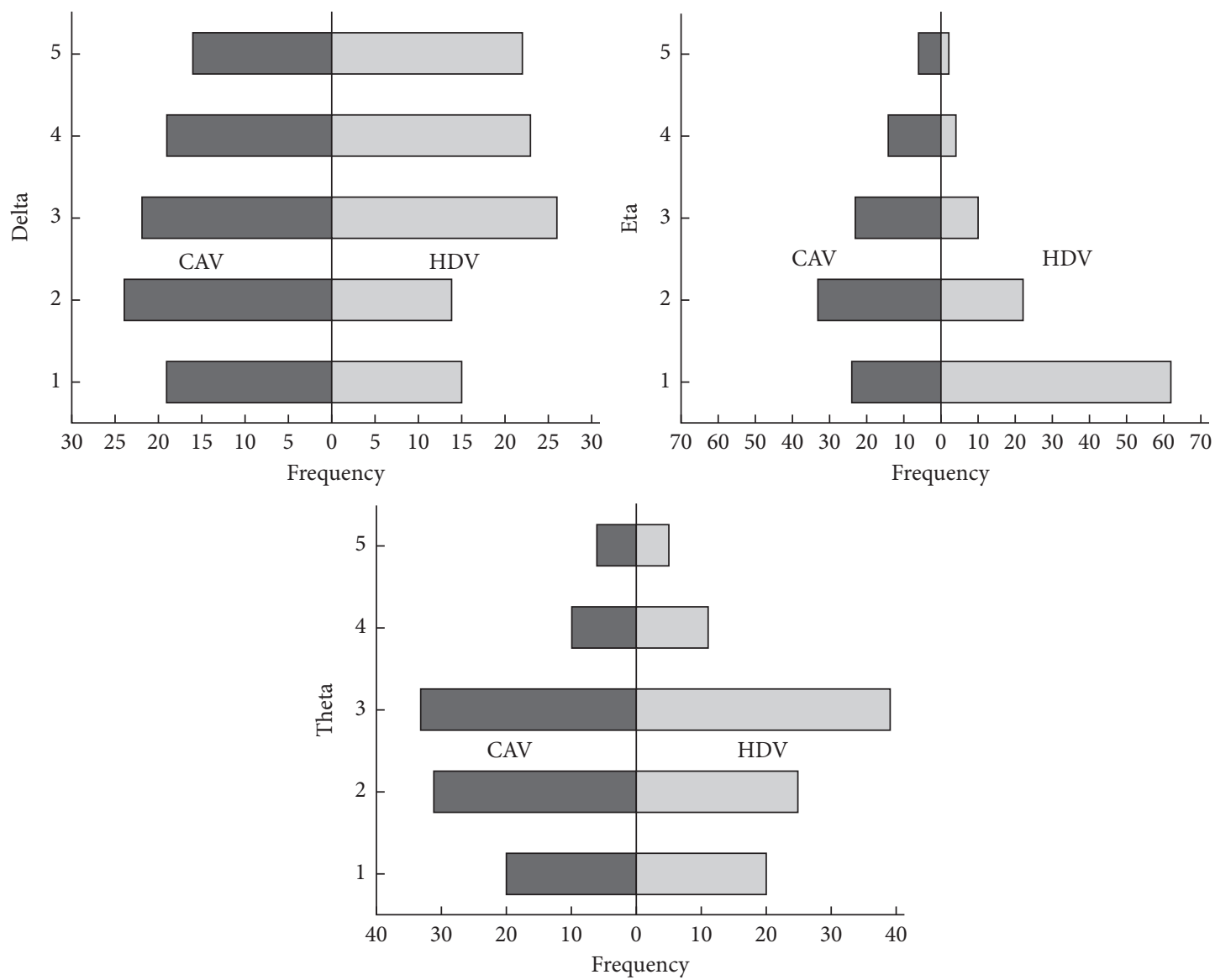

Figure 18: Distributions of the calibrated parameter values of $\delta, \eta, \theta$.
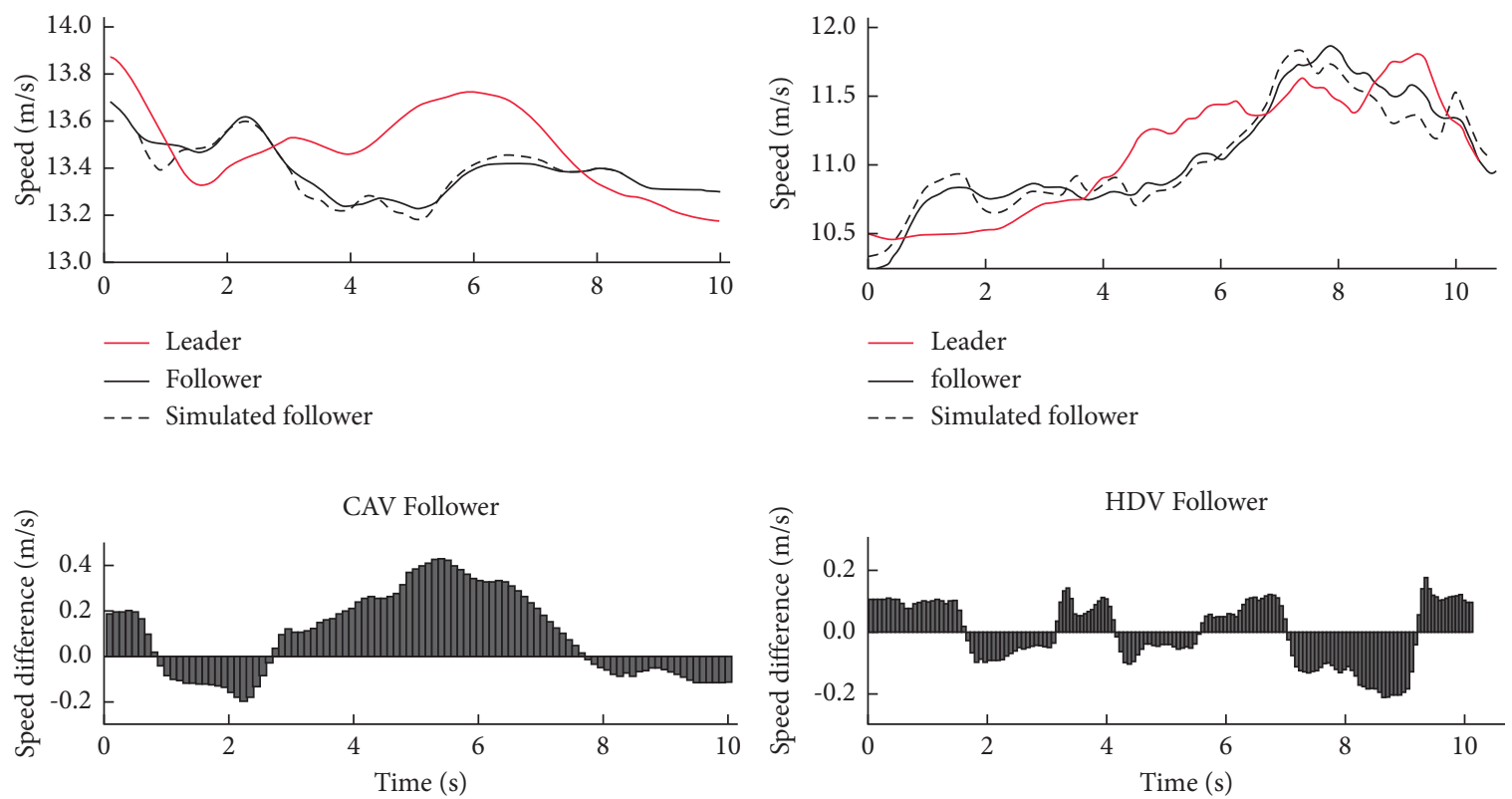

FIgURE 19: Verification for the actual and simulated follower's speed. 


\section{Conclusions and Future Research}

This paper proposes an extended car-following model for mixed traffic in a connected and autonomous driving environment, which can characterize the car-following behavior of CAVs and HDVs in the heterogeneous traffic flow. Using PT, the cooperation between drivers, a human factor, is well integrated into the model. The weight and value functions of PT are modified to simulate the willingness of the follower to cooperate with the front vehicle. To specifically overcome the unavailability of vehicle trajectory data in connected and autonomous vehicle environment, we design and implement a car-following experiment in the CACC scenario and extract vehicle trajectory data based on video recognition. Furthermore, the Lagrangian theory and Kalman filter theory are used to reconstruct the extracted trajectory data to ensure the accuracy of the data.

The model calibration and verification results, combined with the analysis of cooperation for car-following behavior, reveal that the heterogeneity of CAVs and HDVs in mixed traffic is manifested in various ways. Compared with HDVs, CAVs tend to maintain a smaller distance headway with the vehicle in front and have a greater willingness to cooperate with the leader. When the distance headway becomes larger, CAVs are more responsive than HDVs, which is one of the reasons for the more significant fluctuations in the speed and the acceleration of CAVs. It is worth noting that the results reported in this paper are obtained from a relative rather than an absolute perspective. Different results may be obtained depending on the experimental design and the development of CAV technologies.

This paper has several limitations that need further study. One issue is that the experiment is designed without considering intersection signals. The experiment can be improved in the future to study the effect of vehicle-intersection interaction on car-following behavior and some critical traffic flow phenomena, e.g., capacity drop. Another issue is that, when developing the car-following model, only the influence of one vehicle in front is considered. In the future, multiple vehicles in front can be included.

\section{Data Availability}

The data used to support the findings of this study are available from the first author upon request (Shenzhen Ding, 17114228@bjtu.edu.cn).

\section{Conflicts of Interest}

The authors declare that they have no conflicts of interest.

\section{Acknowledgments}

The authors acknowledge that this paper is prepared based on the National Natural Science Foundation of China (NSFC) under grant no. 71871013.

\section{References}

[1] J. Tian, C. Zhu, D. Chen, R. Jiang, G. Wang, and Z. Gao, “Car following behavioral stochasticity analysis and modeling: perspective from wave travel time," Transportation Research Part B: Methodological, vol. 143, no. 2021, pp. 160-176, 2021.

[2] M. Treiber, A. Kesting, and D. Helbing, "Three-phase traffic theory and two-phase models with a fundamental diagram in the light of empirical stylized facts," Transportation Research Part B: Methodological, vol. 44, no. 2010, pp. 983-1000, 2010.

[3] H. S. Mahmassani, "50th anniversary invited article-autonomous vehicles and connected vehicle systems: flow and operations considerations," Transportation Science, vol. 50, no. 4, pp. 1140-1162, 2016.

[4] SAE International in United States, Taxonomy and Definitions for Terms Related to Driving Automation Systems for On-Road Motor Vehicles, 2018, https://saemobilus.sae.org/content/ J3016_201806/.

[5] J. Chen, D. Sun, Y. Li, M. Zhao, W. Liu, and S. Jin, "Humanmachine cooperative scheme for car-following control of the connected and automated vehicles," Physica A: Statistical Mechanics and its Applications, vol. 573, Article ID 125949, 2021.

[6] F. M. Favarò, S. O. Eurich, and S. S. Rizvi, "“Human” problems in semi-autonomous vehicles: understanding drivers' reactions to off-nominal scenarios," International Journal of Human-Computer Interaction, vol. 35, no. 11, pp. 956-971, 2019.

[7] L. A. Pipes, "An operational analysis of traffic dynamics," Journal of Applied Physics, vol. 24, no. 3, pp. 274-281, 1953.

[8] D. C. Gazis, R. Herman, and R. W. Rothery, "Nonlinear follow-the-leader models of traffic flow," Operations Research, vol. 9, no. 4, pp. 545-567, 1961.

[9] M. Treiber, A. Hennecke, and D. Helbing, "Congested traffic states in empirical observations and microscopic simulations," Physical Review E, vol. 62, no. 2, pp. 1805-1824, 2000.

[10] M. Bando, K. Hasebe, A. Nakayama, A. Shibata, and Y. Sugiyama, "Dynamical model of traffic congestion and numerical simulation," Physical Review E, vol. 51, no. 2, pp. 1035-1042, 1995.

[11] P. G. Gipps, "A behavioural car-following model for computer simulation," Transportation Research Part B: Methodological, vol. 15, no. 2, pp. 105-111, 1981.

[12] R. Wiedemann, "Simulation des strassenverkehrsflusses," in Proceedings of the Schriftenreihe des instituts fir Verkehrswesen der Universitiit Karlsruhe, Germany, 1974.

[13] M. Zhu, X. Wang, A. Tarko, and S. e. Fang, "Modeling carfollowing behavior on urban expressways in Shanghai: a naturalistic driving study," Transportation Research Part C: Emerging Technologies, vol. 93, pp. 425-445, 2018.

[14] A. Sharma, Z. Zheng, A. Bhaskar, and M. M. Haque, "Modelling car-following behaviour of connected vehicles with a focus on driver compliance," Transportation Research Part B: Methodological, vol. 126, pp. 256-279, 2019.

[15] L. Xiao and F. Gao, "A comprehensive review of the development of adaptive cruise control systems," Vehicle System Dynamics, vol. 48, no. 10, pp. 1167-1192, 2010.

[16] T. Li, D. Chen, H. Zhou, J. Laval, and Y. Xie, "Car-following behavior characteristics of adaptive cruise control vehicles based on empirical experiments," Transportation Research Part B: Methodological, vol. 147, pp. 67-91, 2021.

[17] G. J. L. Naus, R. P. A. Vugts, J. Ploeg, M. J. G. van de Molengraft, and M. Steinbuch, "String-stable CACC design and experimental validation: a frequency-domain approach," IEEE Transactions on Vehicular Technology, vol. 59, no. 9, pp. 4268-4279, 2010.

[18] V. Milanés, S. E. Shladover, J. Spring, C. Nowakowski, H. Kawazoe, and M. Nakamura, "Cooperative adaptive cruise 
control in real traffic situations," IEEE Transactions on Intelligent Transportation Systems, vol. 15, no. 1, pp. 296-305, 2013.

[19] Z. Yang, Z. Yang, A. Soyoung, W. Meng, and H. Serge, "Stabilizing mixed vehicular platoons with connected automated vehicles: an H-infinity approach," Transportation Research Part B: Methodological, vol. 132, pp. 152-170, 2020.

[20] G. Gunter, C. Janssen, W. Barbour, R. E. Stern, and D. B. Work, "Model-based string stability of adaptive cruise control systems using field data," IEEE Transactions on Intelligent Vehicles, vol. 5, no. 1, pp. 90-99, 2020.

[21] M. Makridis, K. Mattas, A. Anesiadou, and B. Ciuffo, "OpenACC: an open database of car-following experiments to study the properties of commercial ACC systems," Transportation Research Part C: Emerging Technologies, vol. 125, Article ID 103047, 2021.

[22] J. Wang, S. Gong, S. Peeta, and L. Lu, “A real-time deployable model predictive control-based cooperative platooning approach for connected and autonomous vehicles," Transportation Research Part B: Methodological, vol. 128, pp. 271-301, 2019.

[23] Z. Zhong, E. E. Lee, M. Nejad, and J. Lee, "Influence of CAV clustering strategies on mixed traffic flow characteristics: an analysis of vehicle trajectory data," Transportation Research Part C: Emerging Technologies, vol. 115, Article ID 102611, 2020.

[24] W. Zhao, D. Ngoduy, S. Shepherd, R. Liu, and M. Papageorgiou, "A platoon based cooperative eco-driving model for mixed automated and human-driven vehicles at a signalised intersection," Transportation Research Part C: Emerging Technologies, vol. 95, pp. 802-821, 2018.

[25] H. Wang, Y. Qin, W. Wang, and J. Chen, "Stability of CACCmanual heterogeneous vehicular flow with partial CACC performance degrading," Transportmetrica B: Transport Dynamics, vol. 7, no. 1, pp. 788-813, 2019.

[26] A. Ghiasi, X. Li, and J. Ma, "A mixed traffic speed harmonization model with connected autonomous vehicles," Transportation Research Part C: Emerging Technologies, vol. 104, pp. 210-233, 2019.

[27] M. Amirgholy, M. Shahabi, and H. Oliver Gao, "Traffic automation and lane management for communicant, autonomous, and human-driven vehicles," Transportation Research Part C: Emerging Technologies, vol. 111, pp. 477-495, 2020.

[28] V. Milanés and S. E. Shladover, "Modeling cooperative and autonomous adaptive cruise control dynamic responses using experimental data," Transportation Research Part C: Emerging Technologies, vol. 48, pp. 285-300, 2014.

[29] T. Zhang and P. J. Jin, "A longitudinal scanline based vehicle trajectory reconstruction method for high-angle traffic video," Transportation Research Part C: Emerging Technologies, vol. 103, pp. 104-128, 2019.

[30] G. Welch and G. Bishop, "An introduction to the Kalman filter," in Proceedings of the SIGGRAPH, Course, vol. 8, p. 41, Los Angeles, CA, USA, August 2001.

[31] H. Liu, J. Huang, and W. Zhang, "Numerical algorithm based on extended barycentric Lagrange interpolant for two dimensional integro-differential equations," Applied Mathematics and Computation, vol. 396, Article ID 125931, 2021.

[32] M. Montanino and V. Punzo, "Trajectory data reconstruction and simulation-based validation against macroscopic traffic patterns," Transportation Research Part B: Methodological, vol. 80, pp. 82-106, 2015.

[33] K. A. Tversky, "Prospect theory: an analysis of decision under risk," Econometrica, vol. 47, no. 2, pp. 263-291, 1979.
[34] S. E. Shladover, D. Su, and X.-Y. Lu, "Impacts of cooperative adaptive cruise control on freeway traffic flow," Transportation Research Record: Journal of the Transportation Research Board, vol. 2324, no. 1, pp. 63-70, 2012.

[35] J. Sangster, H. Rakha, and J. Du, "Application of naturalistic driving data to modeling of driver car-following behavior," Transportation Research Record: Journal of the Transportation Research Board, vol. 2390, no. 1, pp. 20-33, 2013.

[36] V. Punzo, B. Ciuffo, and M. Montanino, "Can results of carfollowing model calibration based on trajectory data be trusted?" Transportation Research Record: Journal of the Transportation Research Board, vol. 2315, no. 1, pp. 11-24, 2012. 